\title{
Interfacial-curvature-driven coarsening in mass-conserved reaction-diffusion systems
}

\author{
Michio Tateno $\odot^{*}$ and Shuji Ishihara $\odot$ \\ Graduate School of Arts and Sciences, The University of Tokyo, Komaba 3-8-1, Meguro-ku, Tokyo 153-8902, Japan \\ and Universal Biology Institute, The University of Tokyo, Komaba 3-8-1, Meguro-ku, Tokyo 153-8902, Japan
}

(Received 9 October 2020; accepted 11 May 2021; published 11 June 2021)

\begin{abstract}
Mass conservation in chemical species appears in a broad class of reaction-diffusion systems (RDSs) and is known to cause coarsening of the pattern in chemical concentration. Recent theoretical studies on RDSs with mass conservation (MCRDSs) have reported that the interfacial curvature between two states contributes to the coarsening process, which is reminiscent of phase separation phenomena. However, since MCRDSs do not presuppose a variational principle, it is largely unknown whether description of surface tension is operative. In this paper, we numerically and theoretically explore the coarsening process of patterns in MCRDSs in two and three dimensions. We identify the parameter regions where the homogeneous steady state becomes stable, unstable, and metastable. In the unstable region, pattern formation is triggered by usual (type- $\mathrm{II}_{\mathrm{s}}$ ) instability, whereas in the metastable region, nucleation-growth-type pattern formation is observed. In the later stage, spherical droplet patterns are observed in both regions, where they obey a relation similar to the Young-Laplace law and coarsen following the evaporation-condensation mechanism. These results demonstrate that in the presence of a conserved variable, a physical quantity similar to surface tension is relevant to RDSs, which provides insight into molecular self-assembly driven by chemical reactions.
\end{abstract}

DOI: 10.1103/PhysRevResearch.3.023198

\section{INTRODUCTION}

Reaction-diffusion systems (RDSs) are one of the most generic mathematical frameworks that give rise to spatiotemporal patterns and have been applied to various phenomena, ranging from physics, chemistry, biology, and geology to ecology [1-4]. The Turing mechanism explains how a homogenous initial state is destabilized to develop a spatial structure; infinitesimally small fluctuations with a particular range of wave number $(0<) q_{-}<q<q_{+}$grow exponentially, which is known as type- $\mathrm{I}_{\mathrm{s}}$ instability [1]. Despite this analysis being valid only for every early linear time regime, the wave number with the fastest growth $q_{\mathrm{m}}$ usually provides a good estimation of the characteristic length scale $\ell_{\mathrm{m}} \equiv 2 \pi / q_{\mathrm{m}}$ for final steady-state patterns, such as stripes or hexagonal dots in two-dimensional (2D) systems.

Mass-conserved reaction-diffusion systems (MCRDSs) do not follow this trend. MCRDSs were originally developed to model molecular localization of membrane-bounded proteins inside cells, specifically Rho-family GTPases, which regulate cell polarity [5-15]. In such systems, although the instability of a homogenous solution is evaluated via the same linear stability analysis as in the Turing mechanism, the region of the unstable modes is large scale (i.e., $q_{-}=0$; type- $\mathrm{II}_{\mathrm{s}}$ instability [1]). Notably, after a linear time regime, the characteristic length scale of the patterns

\footnotetext{
*c-tateno@g.ecc.u-tokyo.ac.jp

Published by the American Physical Society under the terms of the Creative Commons Attribution 4.0 International license. Further distribution of this work must maintain attribution to the author(s) and the published article's title, journal citation, and DOI.
}

easily grows beyond $\ell_{\mathrm{m}}$ and often reaches a length scale comparable to the system size $L$; coarsening of patterns occurs even though energy variational principle is not assumed. In the context of biomolecular self-assembly, this feature indicates that proteins accumulate at one site, not spreading over multiple sites, and is crucial to determining the unique directionality of cells. It was also discussed that the resulting scalability of the pattern against the system size has biological significance in morphogenesis [16-21].

Pattern formation of MCRDSs in one dimension has been intensively investigated in previous research. Two types of MCRDSs were mainly studied: in the first case, referred to as "Turing type" [11], multiple peaklike domains, illustrated in the left panel of Fig. 1(a), appear from a homogeneous initial state via usual (type- $\mathrm{II}_{\mathrm{s}}$ ) instability $[5,6]$; the second case is "wave-pinning type" [8], where the system is bistable with high and low chemical concentration states, and by exposing a finite (not infinitesimally small) perturbation to the homogeneous state, mesalike concentration profiles emerge as illustrated in the right panel of Fig. 1(a). Both systems exhibit coarsening, i.e., smaller peaks and mesas shrink and disappear while larger ones grow. For the Turing type, both the height and width of the peak increase, whereas for the wave-pinning type, only the width of the mesa grows while keeping the height [see Fig. 1(a)]. For a long timescale, the system reaches a single isolated domain in the Turing type. On the other side, for the wave-pinning type, the decrease in the number of domains significantly slows down at some point, resulting in the apparent coexistence of multiple domains.

Recent studies revealed that the above differences between the Turing type and the wave-pinning type would be rather superficial $[11,12,14]$. Chiou et al. [11] found that the peaklike profile in Turing-type MCRDSs is owing to the unsaturation of the peak height. That is, the peaklike pattern is transient and 
(a)
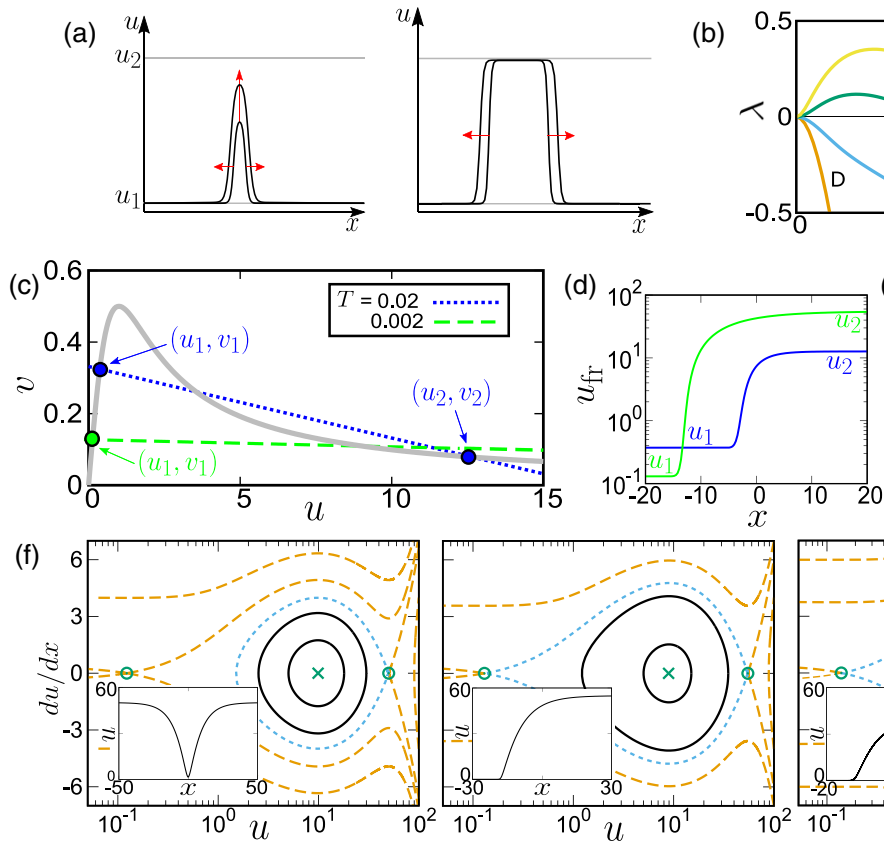

(b)

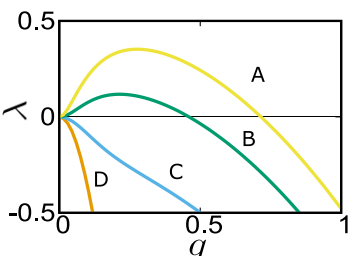

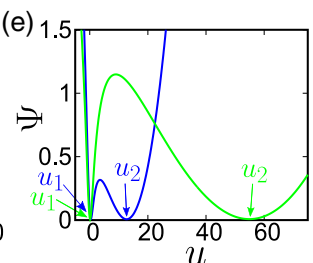

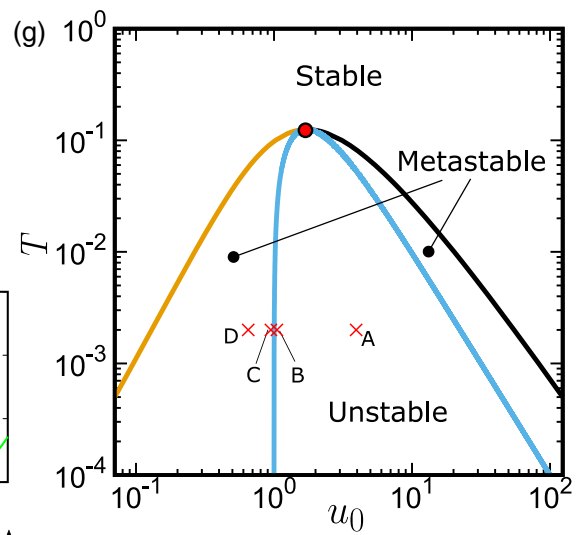

(h)

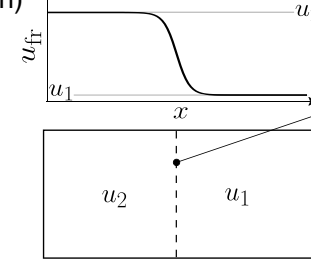

$u_{2}$

curved interface

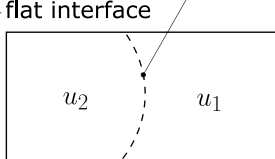

FIG. 1. Steady-state analysis of MCRDSs in one dimension. (a) Two typical concentration profiles in MCRDSs. $u_{1}$ and $u_{2}$ denote $u$ at the bistable points. Depending on whether $u$ in the domain is saturated to the stable points or not, the concentration profile of $u$ show mesalike (wave-pinning type, right panel) or peaklike (Turing type, left panel) behavior. (b) Dispersion relation for the homogeneous state. The growth rate $\lambda$ is shown for various parameter values indicated by the labels corresponding to those in (g). For large scale $(q \rightarrow 0), \lambda$ follows $\lambda=-\left(D_{v} f_{u}-D_{u} f_{v}\right) /\left(f_{u}-f_{v}\right) q^{2}+O\left(q^{4}\right)$, and the sign of the prefactor of $q^{2}$ determines the stability of the homogeneous state. (c) Mapping of the steady stable points on the $u-v$ space for two different parameter values of $T$. The gray curve is the nullcline for the reaction term $f$. The blue and green lines represent $p_{\infty}=D_{u} u+D_{v} v$ for $T=0.02$ and 0.002 , respectively. The blue and green circles represent the stable fixed points. Note that $f$ is independent of the choice of the model parameters in our normalization (see Appendix B). (d) Steady-front solutions $u=u_{\mathrm{fr}}$ for $T=0.02$ and 0.002 . (e) Function profiles of $\Psi\left(u, p_{\infty}\right)$ for $T=0.02$ and 0.002 . (f) The trajectories of the steady solutions $(u, d u / d x)$ for three different conditions of $p=60$ (left), $p=p_{\infty} \sim 64$ (middle), and $p=70$ (right). Here $T$ is set as 0.002 . The black solid, blue dotted, and brown dashed lines represent periodic, localized, and unphysical solutions, respectively. The function profiles of $u(x)$ for localized solutions are shown in the insets. Note that the inset of the middle panel corresponds to the steady front solution $u_{\mathrm{fr}}$. The green circle and cross symbols represent stable and unstable fixed points, respectively, and the former corresponds to $\left(u_{1}, 0\right)$ and $\left(u_{2}, 0\right)$. (g) Phase diagram. The blue curve represents the border of the unstable region where type- $\mathrm{II}_{\mathrm{s}}$ instability is operative. The brown and black curves represent the set of stable points, $u_{1}$ and $u_{2}$, respectively. We denote the region outside of the two curves as stable and regions that are neither stable nor unstable as metastable. The red circle symbol is a critical point, $\left(u_{\mathrm{c}}, T_{\mathrm{c}}\right)=(\sqrt{3}, 1 / 8)$. The red cross symbols labeled A-D represent parameter sets with $T=0.002$ and $u_{0}=3.94,1.05,0.95,0.65$, respectively. (h) A steady front solution $\left(u_{\mathrm{fr}}, p_{\infty}\right)$ obtained via $1 \mathrm{D}$ analysis corresponds to a solution in which two stable states are segregated by a flat interface in higher dimensions (left). For an interface with positive mean curvature (right), $p$ takes a larger value than $p_{\infty}$, which plays a central role in long-time coarsening dynamics.

it will eventually become mesalike for a sufficiently large system size and long simulation time. Their study also discussed that the difference in the peak heights causes a difference in "recruitment power", which enhances the flux of the chemical component from lower peaks to higher ones (see $\nabla p$ introduced in Sec. II). This drives faster coarsening of peaks seen in the Turing type than the wave-pinning type. Furthermore, type- $\mathrm{II}_{\mathrm{s}}$ instability can occur in the wave-pinning type MCRDSs by choosing appropriate parameter values [10-12]. Taken together, it is understood that there is a quantitative but no essential difference between long-term dynamics of two cases. We note that the coexistence of multiple peaks can also be induced by a slight violation of mass conservation [12,14] or the introduction of negative feedback [12].

However, since earlier studies have conducted intensive analyses only in 1D systems, there remains a fundamental untested component in understanding the dynamics of MCRDSs. For higher dimensions, the interfacial morphology of patterns may be crucial to the pattern formation dynamics of MCRDSs. Indeed, Refs. [11,12] reported that small dropletlike domains tend to disappear whereas large ones tend to grow, which reminds us of the evaporation-condensation, or Lifshitz-Slyozov-Wagner (LSW) process in phase separation phenomena [22-30]. The similarity in phase diagram behaviors between MCRDSs and phase separation systems $[10,12,13]$ also supports this analogy. However, in MCRDSs, the chemical reaction violates the detailed balance condition in general. Although several studies [13,31] considered the mapping from MCRDSs to a universal model of phase separation (i.e., the Cahn-Hilliard model), the existence of the variational functional, which is a prerequisite for the theory of phase separation, is not obvious. In particular, it remains elusive whether the physical description of the surface tension, which plays a prominent role in the LSW process, could be rationalized for MCRDSs, because a detailed investigation of MCRDSs in 2D and 3D has not been conducted so far. 
Motivated by the question raised above, this study explores the influence of curved interface on pattern formation dynamics in MCRDSs. We perform extensive numerical simulations using multiple GPUs for both 2D and 3D systems. The obtained dynamics are analyzed using methods developed for phase separation phenomena, together with technique of dynamical systems theory. By investigating the similarities and differences in coarsening process between MCRDSs and phase separation systems, we explore whether or not a concept of surface tension is relevant to MCRDSs.

\section{SYSTEM DESCRIPTION}

We consider a system composed of two chemical species, $U$ and $V$, and denote their concentration fields as $u(t, \boldsymbol{r})$ and $v(t, \boldsymbol{r})$, respectively. These species are interconvertible by chemical reactions $U \rightarrow V$ and $V \rightarrow U$, the rates of which are $k_{u} u$ and $k_{v} v$, respectively. Note that these two reactions do not have to be in reverse relationship and the system may not satisfy detailed balance. Both the transition rates $k_{u}$ and $k_{v}$ can be dependent on the concentration states $u$ and $v$, as a result of positive feedback regulations in the chemical reaction network. Moreover, both $U$ and $V$ migrate over space by thermal diffusion. We model the time-evolution equations for $u$ and $v$ as the following reaction-diffusion equations:

$$
\begin{aligned}
& \frac{\partial u}{\partial t}=D_{u} \nabla^{2} u-f(u, v), \\
& \frac{\partial v}{\partial t}=D_{v} \nabla^{2} v+f(u, v) .
\end{aligned}
$$

Here, $D_{u}$ and $D_{v}$ are the diffusion coefficients of $U$ and $V$, respectively, and we assume $D_{u}<D_{v} . f$ is the reaction term and written as $f=k_{u} u-k_{v} v$. By adding the above two equations, we obtain

$$
\frac{\partial s}{\partial t}=\nabla^{2} p
$$

where $s=u+v$ and $p=D_{u} u+D_{v} v$. Equation (3) indicates that $s$ is a conserved variable without material sources, and transported from large to small $p$ region. We show later that $p$ plays a role similar to that of chemical potential in the coarsening regime.

The relevance of adopting MCRDSs to describe cellular processes can be rationalized as follows: (i) Many subcellular processes, such as cell polarity formation, are sufficiently rapid ( $\sim 1 \mathrm{~min})$ compared with the production and degradation rates of the molecules $(>10 \mathrm{~min})$. Such processes are usually conducted only through the activation and deactivation of molecules, such as phosphorylation and dephosphorylation. The ratio of molecules between "on" and "off" states changes; however, the total mass of the molecule is conserved throughout the processes. (ii) Molecular interaction contains positive feedback loops that regulate the switching rate between molecular states, e.g., the acceleration of phosphorylation is found in subcellular processes. (iii) Molecular states are often associated with an affinity to the cellular membrane; thus, molecules in different states are likely to attach to or detach from the membrane. As a result, molecular diffusion coefficients are significantly different between the molecular states, being small on the cell membrane but large in the cytosol. As these processes are basic, many previous models, explicitly or implicitly, contain chemical components that are conserved through dynamics. In Ref. [5], it was shown that in a model with these conditions, a uniform distribution of molecules can be destabilized by the type- $\mathrm{II}_{\mathrm{s}}$ instability, leading to an accumulation of molecules at one site.

To examine the generality of the results presented below, we employ two models, following earlier works on MCRDSs, both of which violate the detailed balance condition. Model I employs $k_{u}=c a /\left(b+u^{2}\right)$ and $k_{v}=c$ [5,6]. For model II, $k_{u}=\alpha$ and $k_{v}=k_{0}+\beta u^{2} /\left(K^{2}+u^{2}\right)$ are employed [8]. The former and latter models correspond to the Turing type and wave-pinning type, respectively, by the criteria mentioned in the Introduction [11]. Because these models share many aspects in their dynamical trends, we primarily describe the results of model I in the main text and make comments when there are remarkable differences in the results between the two models. The results from model II are provided in Appendix E.

In the results presented below, the statistical data are based on simulations performed on $4096^{2}$ and $1024^{3}$ grids for 2D and 3D systems, and have normalized units, described in Appendix B. After normalization, the governing Eqs. (1) and (2) have two free parameters: One is associated with the reaction term and is defined as $\mathcal{R}=a / b$ and $\alpha / \beta$ for models I and II, respectively; the other is $\mathcal{D}=D_{v} / D_{u}$, associated with the diffusion constants. Following Refs. [6,11], we set $\mathcal{D}=50$ and 100 for models I and II, respectively. As we will show below, the stability of the homogeneous state is determined by the product of these parameters $T^{-1} \equiv \mathcal{R D}$, and the initial chemical concentrations that determine the conserved quantity in the system.

\section{RESULTS}

\section{A. Phase diagram of MCRDSs}

Before investigating pattern formation dynamics in MCRDSs, we begin by identifying their parameter dependencies by analyzing the initial homogeneous and eventual bistable solutions. The results in this subsection are mainly obtained by the analysis in 1D systems.

We first consider the stability of the homogeneous steady solution $\left(u_{0}, v_{0}\right) . u_{0}$ and $v_{0}$ are determined by the conditions $f\left(u_{0}, v_{0}\right)=0$ and $s_{0}=u_{0}+v_{0}$, where $s_{0} \equiv \int s d \boldsymbol{r} / V$ is the mean molecular concentration conserved through the dynamics [see Eq. (3)], and $V$ is the volume of the system. We suppose that the fixed point $\left(u_{0}, v_{0}\right)$ is linearly stable when the diffusion terms are absent in Eqs. (1) and (2) (otherwise the system runs to infinity). This is conditioned by $f_{v}-f_{u}<0$, where $f_{u}$ and $f_{v}$ represent $\partial f(u, v) / \partial u$ and $\partial f(u, v) / \partial v$ at $\left(u_{0}, v_{0}\right)$, respectively. In the presence of the diffusion terms, the homogeneous state becomes linearly unstable against an infinitesimally small perturbation with wave number $q$ between $0<q^{2}<\left(D_{u} f_{v}-D_{v} f_{u}\right) / D_{u} D_{v}$ when $\left(D_{v} f_{u}-D_{u} f_{v}\right) /\left(f_{u}-f_{v}\right)<0$ is satisfied. Since the denominator is positive as mentioned above, the instability condition is simply written as

$$
D_{v} f_{u}-D_{u} f_{v}<0 .
$$

For this condition to hold for model I, $T<T_{\mathrm{c}} \equiv 1 / 8$ is necessary (see Appendix B). The dispersion relation obtained in 
the analysis is shown in Fig. 1(b), where $\lambda(q)$ represents the growth rate of the perturbation with wave number $q$. Note that, owing to mass conservation, $\lambda(q)$ always starts from zero unlike the cases of typical RDSs. Thus, the condition of Eq. (4) does not belong to the standard Turing [or type-I stationary $\left(\mathrm{I}_{\mathrm{s}}\right)$ ] or Hopf [type-III oscillatory $\left(\mathrm{III}_{\mathrm{o}}\right)$ ] instability but is classified as type-- $\mathrm{II}_{\mathrm{s}}$ instability [1].

Next, we explore the dynamic behavior in the long time regime. The most significant feature in MCRDSs is the existence of a conserved variable $s$. The transport of $s$ is driven by the spatial gradient of a scalar variable $p$ as indicated by Eq. (3). At the steady state, $p$ satisfies $\nabla^{2} p=0$. This implies that $p$ becomes a constant value in the absence of material source. Owing to the constant nature of $p,(u, v)$ is constrained on a line $p=D_{u} u+D_{v} v$ which has three intersection points with the nullcline $f=0$ [see Fig. 1(c)]. Two of them, indicated by $\left(u_{1}, v_{1}\right)$ and $\left(u_{2}, v_{2}\right)\left(u_{1}<u_{2}\right)$ in the figure, are locally stable points, to which $(u, v)$ tends to relax. Therefore, it is expected that the system behaves similar to a bistable system, where the bistability is induced by the diffusion rate asymmetry between components $U$ and $V$.

We then consider a steady front solution $u=u_{\mathrm{fr}}(x, t)$, where two stable states $u_{1}$ and $u_{2}$ are connected via a single interface [see Fig. 1(d)]. Note that the asymptote of the front solution $u_{\mathrm{fr}}(x)$ shows exponential decay on both sides of the interface, indicating that the width of the interface is finite and thus well-defined. We denote the corresponding value of $p$ as $p_{\infty}$. The form expected for the front solution is $u_{\mathrm{fr}}(x-c t)$; it either translates with a constant speed $c$ while maintaining the concentration profile or stays at the same positions (i.e., $c=0$ ) [32]. According to earlier studies [5-14], the latter static solution is typical in MCRDSs. Indeed, in Appendix C, we find that $c=0$ is the only compatible solution. We obtain this solution when

$$
\Psi\left(u_{1}, p_{\infty}\right)=\Psi\left(u_{2}, p_{\infty}\right)
$$

is satisfied, where $\Psi(u, p)$ is defined by

$$
\Psi(u, p)=\int^{u} f\left(u,\left(p-D_{u} u\right) / D_{v}\right) d u .
$$

Here, $u_{1}$ and $u_{2}$ coincide with two minima of $\Psi\left(u, p_{\infty}\right)$ and the condition Eq. (5) determines the value of $p_{\infty}$ (see Appendix C). Hence, a front solution for the conserved variable $s$ is given by $s_{\mathrm{fr}}(x)=\left[\left(D_{v}-D_{u}\right) u_{\mathrm{fr}}(x)+p_{\infty}\right] / D_{v}$. Examples of $\Psi(u)$ at $p=p_{\infty}$ are shown in Fig. 1(e) for $T=0.02$ and 0.002 , where the two minima correspond to the bistable fixed points $u_{1}$ and $u_{2}$. The figure shows that the smaller the value of $T$, the larger the difference between the two points. This increases the time duration required for saturation to the fixed points and results in the transient peaklike profile as shown in the left panel of Fig. 1(a). Similar patterns are also observed in the phase separation systems where the shape of double-well potential is highly asymmetric (for example, see Refs. [25,27$30,33,34])$.

The front solution $\left(u_{\mathrm{fr}}, p_{\infty}\right)$ is a special one among the static stationary solutions of Eqs. (1) and (2). We can obtain a family of the static solutions by solving an ordinary differential equation $D_{u} d^{2} u / d x^{2}=f\left(u,\left(p-D_{u} u\right) / D_{v}\right)$, where $p$ is constant. Figure 1(f) shows the trajectories of the solutions $(u, d u / d x)$ for three different conditions of $p<p_{\infty}$, $p=p_{\infty}$ and $p>p_{\infty}$. The black solid lines represent periodic solutions. The brown dashed lines are unphysical since $u(x)$ diverges for $|x| \rightarrow \infty$. The blue dotted lines provide localized solutions; for $p<p_{\infty}$ and $p>p_{\infty}$, they correspond to homoclinic orbits in $(u, d u / d x)$ space, and have holelike and peaklike profiles in $u(x)$, as shown in the insets of the right and left panels, respectively. A heteroclinic orbit exits only when $p=p_{\infty}$, and yields the front solution $u_{\mathrm{fr}}(x)$ (see the inset of the middle panel). We mention that a similar structure for a family of static solutions is found in phase separation phenomena with an asymmetric free energy [34].

The phase diagram of the MCRDSs raised by the above analysis is shown in Fig. 1(g). In the figure, the horizontal and vertical axes represent the composition of $u$ at the initial homogeneous state and the dimensionless parameter $T$, respectively. The region enclosed by the blue curve is obtained using the instability condition Eq. (4); hereafter, we refer to this region as the unstable region. The brown and black curves represent the sets of two stable states $u_{1}$ and $u_{2}$, respectively. We call the region between the two curves a metastable region. As we show later, in this region, homogeneous states are stable in a deterministic sense; however, inclusion of the concentration fluctuation to MCRDSs provokes pattern formation. The phase diagram can also be determined by $\Psi\left(u, p_{\infty}\right)$; the condition that the second derivative is negative, $d^{2} \Psi / d u^{2}=\left(D_{v} f_{u}-D_{u} f_{v}\right) / D_{v}<0$, agrees with Eq. (4), and the two minima of $\Psi$ coincide with two stable states $u_{1}$ and $u_{2}$. However, although $\Psi$ exhibits a similarity to a bulk free energy in phase separation phenomena, MCRDSs cannot be written in a variational form without any approximation procedures in general [13,31].

In the following subsections, we will study pattern formation dynamics of MCRDSs in 2D and 3D, separately in the unstable and in the metastable regions. In higher dimensions, the front solution $\left(u_{\mathrm{fr}}, p_{\infty}\right)$ obtained above is for a solution in which two stable states are segregated by a flat interface [see the left panel of Fig. 1(h)]. We will see that $u_{\mathrm{fr}}$ plays a pivotal role in long-time coarsening dynamics; $p$ for a curved interface (see the right panel) is larger than $p_{\infty}$, which is responsible for material transport among dropletlike domains, leading to the evaporation-condensation (LSW) coarsening law.

Finally, we make three remarks on the above steady-state analysis. First, in two-component MCRDSs, coarsening of patterns is broadly observed [5-14] when nullcline $f(u, v)=$ 0 has two stable intersections with $p=D_{u} u+D_{v} v$, where $p$ is constant. Dynamics other than coarsening such as a traveling wave and a turbulent pattern are also possible in the presence of multiple conserved variables (see Refs. [35,36], for instance). Second, a relation equivalent to Eq. (5) and a method to construct a phase diagram based on this relation were proposed in a recent paper by Brauns et al. [13], in which the reaction-term dependence of the phase diagrams is discussed in detail. Both their and our approaches do not require any approximations, and thus the resulting phase diagram [Fig. 1(g)] improves on those presented in previous studies (see, e.g., Refs. [10,12]). Third, in phase separation systems, there exists a region inside the spinodal lines where the growth rate for steady periodic solutions is larger than that for steady homogenous solutions (known as the nucleationdominated region in Refs. [33,34,37]), which can modify the 

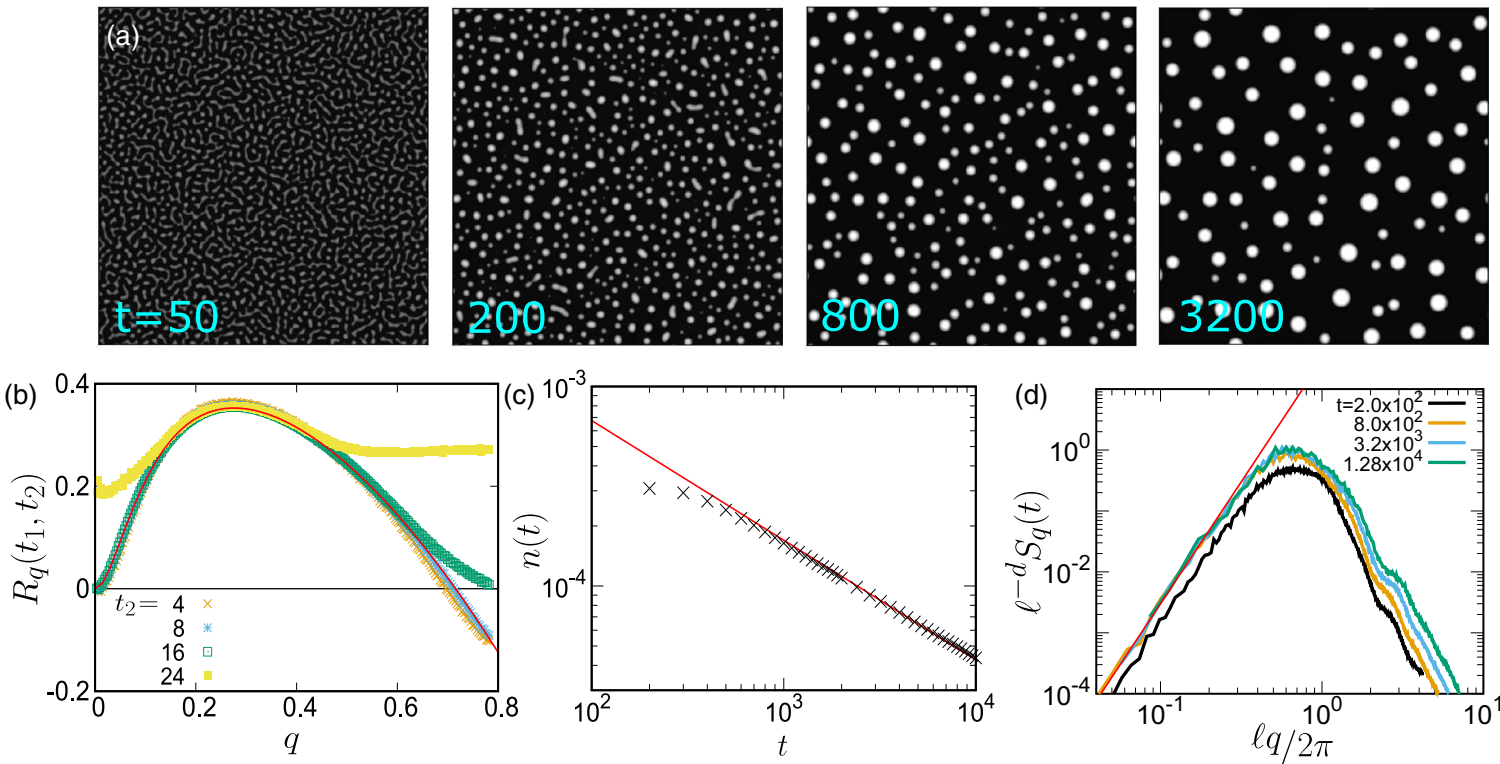

FIG. 2. Pattern formation in the unstable region. (a) Formation process of the droplet structure. Spatial patterns of $s=u+v$ at $t=$ $50,200,800$, and 3200 are shown. The system size is $1024^{2}$. (b) Growth rate measured by simulation results via a relation, $R_{q}\left(t_{1}, t_{2}\right)=$ $\frac{1}{2\left(t_{2}-t_{1}\right)} \ln \left(S_{q}\left(t_{2}\right) / S_{q}\left(t_{1}\right)\right)$, where $S_{q}(t)$ is a structure factor at time $t$. In this figure, $t_{1}$ is fixed as $t_{1}=2$, and the results for various time $t_{2}$ $(=4,8,16,24)$ are shown. The red curve represents a theoretical prediction of growth rate in the linear regime. (c) Temporal changes in the number of droplets per unit volume, $n(t)$. The red line represents a power-law function with exponent 0.60 . (d) Scaled structure factor $\ell^{-d} S(q, t)$ (where $d$ is the spatial dimension) for various times. Here, $\ell(t)$ is a length scale determined by $\ell^{d} n=1$, a typical center-of-mass distance among neighboring droplets. The red line represents a power function with exponent 4 . The above data are obtained by simulations using parameter set A indicated in Fig. 1(g).

phase diagram. In Appendix D and Fig. 6, we show that a similar region also exists in MCRDSs.

\section{B. Pattern formation in the unstable region}

We first study the unstable region. To focus on similarities and differences between pattern formation in MCRDSs and phase separation systems, in the following section, the time evolution of the conserved variable $s$ is investigated. Figure 2(a) shows 2D pattern formation process of $s$ for parameter set A, indicated in Fig. 1(g). An initial pattern with a characteristic length spontaneously appears everywhere in space $(t=50)$. Then, elongated and branched domains are relaxed to spherical shape $(t=200)$. Using linear stability analysis, the deviation of $s$ from the initial homogeneous concentration $s_{0}, \delta s=s-s_{0}$, is expected to obey $\delta s(\boldsymbol{q}, t) \sim$ $\delta s(\boldsymbol{q}, 0) \exp (-\lambda(q) t)$, where $\boldsymbol{q}$ is the wave number. $\lambda$ is the maximum growth rate of the perturbation with the wave number $\boldsymbol{q}$ [see also Fig. 1(b)], which can be estimated from the simulation results via $R_{q}\left(t_{1}, t_{2}\right)=\frac{1}{2\left(t_{2}-t_{1}\right)} \ln \left(S_{q}\left(t_{2}\right) / S_{q}\left(t_{1}\right)\right)$, where $S_{q}(t)=\langle s(\boldsymbol{q}, t) s(-\boldsymbol{q}, t)\rangle$ is the structure factor at time $t$ and the bracket represents the average over the angle. If $R_{q}\left(t_{1}, t_{2}\right)$ is independent of time, $R_{q}$ is to be equivalent to $\lambda(q)$. Figure 2(b) shows the growth rate measured by the simulation results, where $R_{q}$ at different times collapse onto $\lambda(q)$ estimated by linear analysis (see the red curve) for a short time. This indicates that the observed pattern formation seen is driven by type- $\mathrm{II}_{\mathrm{s}}$ instability.

In the later stage $(t \geqslant 400)$, spherical dropletlike domains coarsen over time. More specifically, the smaller droplets tend to shrink and eventually disappear, whereas the larger droplets tend to grow, see supplemental movie [38]. This trend is reminiscent of the evaporation-condensation (LSW) mechanism in phase separation phenomena as pointed out in Refs. [11,12,14]. To qualitatively characterize this growth behavior, we track how the number of droplets changes over time. Figure 2(c) shows the number density of the droplets $n(t)$, where $n$ shows a power-law decay for the long time regime. The estimated power-law exponent is approximately 0.60 [see red line in Fig. 2(c)]. Because the volume (area) occupied by a single droplet is roughly $n^{-1}$, the characteristic length scale at time $t$ is estimated as $\ell=n^{-1 / d}(d$ being spatial dimension, i.e., $d=2$ ), we accordingly obtain $\ell \propto t^{-0.30}$. This power-law exponent, often called the growth exponent, takes a different value depending on the coarsening mechanism. The value of 0.30 observed here is close to the growth exponent known for the LSW mechanism (1/3). We perform the same analysis for a 3D simulation at the same concentration (i.e., for parameter set A in Fig. 1(f)) and the obtained growth exponent is 0.29 . These results are consistent with the fact that the growth exponent in the LSW mechanism is independent of dimensionality for $d \geqslant 2$.

In phase separation phenomena, the power-law decay in the characteristic length is a consequence of a self-similar growth in the patterns. To examine whether this is the case for our problem, we perform a dynamic scaling analysis for $S_{q}$. In Fig. 2(d), we show that $g \equiv \ell(t)^{-d} S_{q}(t)$ as a function of $\ell q$. When a self-similar growth is the case, all the structure factors at different times should be mapped onto a unique mater curve. In this figure, we can observe that $g$ for small $q$ 

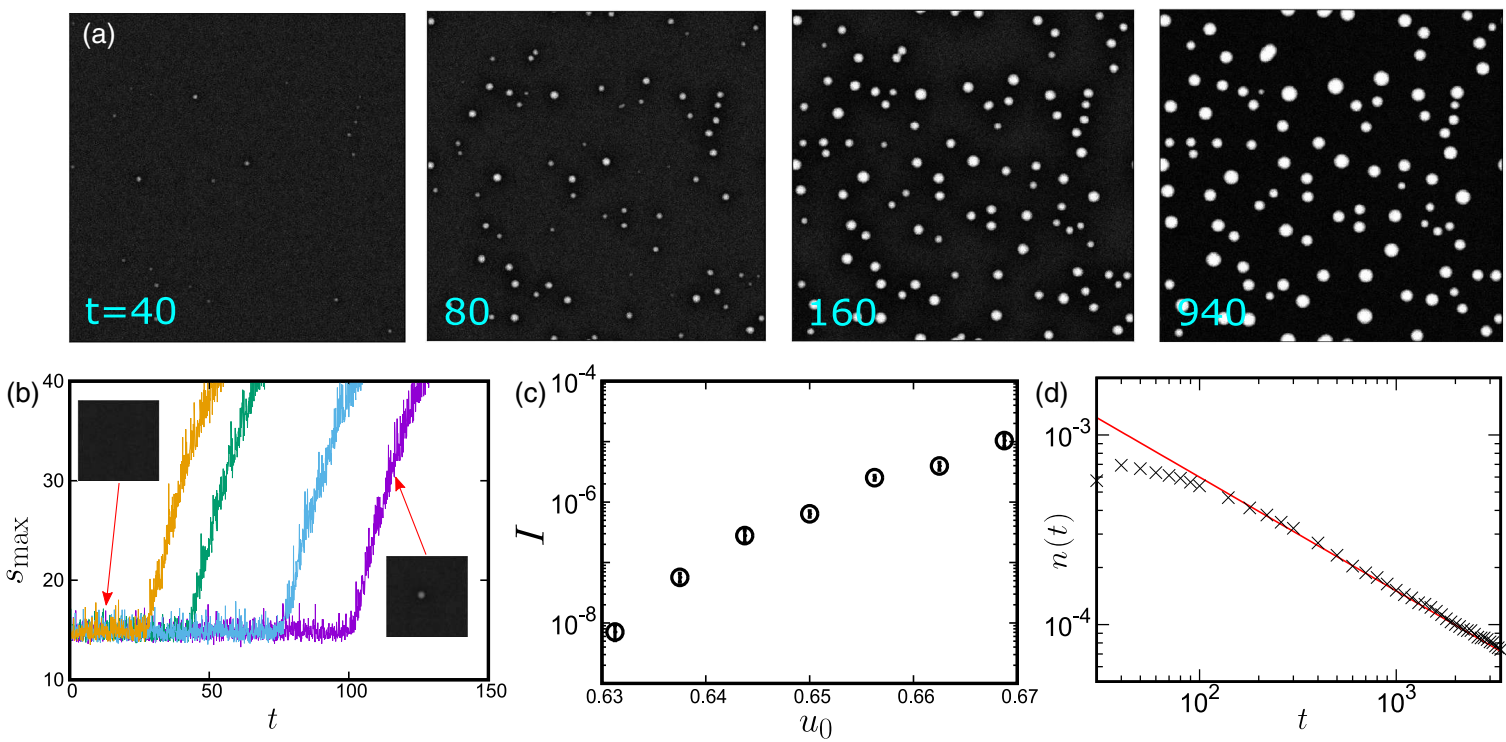

FIG. 3. Nucleation-growth-type pattern formation in the metastable region. (a) Formation process of droplet structure for parameter set D $\left(u_{0}=0.65, T=0.002\right.$ ). Spatial patterns of $s=u+v$ at time $t=40,80,160$, and 940 are shown; the system size is $1295^{2}$. (b) Time series of the maximum value of $s, s_{\max }$, for four independent simulations using parameter set D. The sudden increase in $s_{\max }$ at random time indicates that the formation of droplets is a stochastic activation process. The system size is chosen to be $162^{2}$, which is small enough to observe a single droplet formation in homogeneously mixed space, and large enough compared to the size of the nucleus. (c) Nucleation rate under various initial conditions. (d) Temporal changes in the number of droplets per unit volume $n(t)$ for parameter set $\left(u_{0}=0.70, T=0.002\right)$. The red line represents a power-law function with exponent 0.60 .

collapses onto a single power-law function after the formation of spherical droplets $(t \geqslant 400)$, indicating that the distance between center-of-mass positions of neighboring droplets are scaled by a unique length $\ell$. On the other side, for large $q$, $S_{q}$ slowly shifts toward the higher- $q$ side for intermediate time regime (e.g., $t \sim 3200$ ), meaning that the concentration profile of droplets is not completely scaled by $\ell$ in this time regime. This is because $s$ inside some droplets is not saturated to the stable fixed point $s_{2}$. We mention that such breakdown of self-similarity originating from unsaturation is not specific to MCRDSs, but is also observed in the phase separation systems when the shape of double-well potential is highly asymmetric (for example, see Refs. [25,27-30,33,34]).

For low- $q, S_{q}$ is proportional to $q^{4}$, which is often observed in phase transition phenomena with a conserved order parameter (see, e.g., Refs. [39-41]). According to the literature, this trend appears in the case where surface tension plays a central role in the coarsening process rather than thermal fluctuation [42] and the phase separating structure is statistically isotropic [43].

\section{Pattern formation in the metastable region}

Next, we focus on pattern formation dynamics in the metastable region. We confirm from simulations that the homogeneous state is destabilized for parameter sets in the unstable region [A and $\mathrm{B}$ in Fig. $1(\mathrm{~g})$ ] but is stable in the metastable region $(C$ and $D)$, which is consistent with the analysis performed in Sec. III A. However, by choosing appropriate nonhomogeneous initial conditions (while the mean concentration is kept constant in the metastable region), we find cases where systems relax to a bistable spatial struc- ture. To see typical kinetics in the metastable region, in this section we investigate how patterns develop in the presence of concentration fluctuation. We incorporate intrinsic fluctuations originating from both diffusion and chemical reactions, referring to Ref. [44], see Appendix A for details.

Figure 3(a) shows the time evolution of conserved variable $s$. In the early stage $(t=40,80)$, droplets appear at random positions and at stochastic timing. The size of the droplets increases with time. At the intermediate time $(t=160)$, the growth of the droplets significantly slows down. This is because molecules in the region surrounding the droplets are almost exhausted, where $s$ is in the vicinity of the minimum stable point. For a droplet to grow further, it is necessary to transport material from other droplets. Indeed, at the later stage $(t=940)$, the growth of the droplets is accompanied with shrinkage and eventual disappearance of small droplets. These pattern-formation dynamics are similar to those in the nucleation-growth-type phase separation of pure fluids, multicomponent liquid mixtures, and alloys [45] but differs from those in so-called stochastic Turing patterns, which are induced by stochastic resonance [44,46-50].

As an indicator of the formation of the nucleus, we monitor the time evolution of a maximum concentration value of $s_{\max }$. The results obtained by four independent simulations are shown in Fig. 3(b). We can see that $s_{\max }$ initially fluctuates around a particular value and suddenly starts to increase. The onset time $t_{\text {inc }}$ clearly indicates the birth of nucleus. From the onset time, we compute the nucleation rate $I$ via $I=N /\left\langle t_{\text {inc }}\right\rangle V$, where $N$ is the number of nuclei at time $t_{\text {inc }}, V$ is the volume (area), and the bracket represents the ensemble average. Sixteen independent simulations were performed to determine $I$ under various initial 
(a)

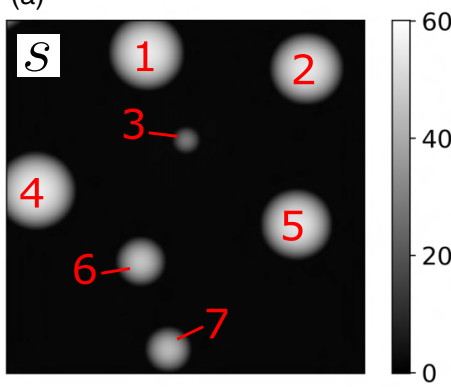

(b)

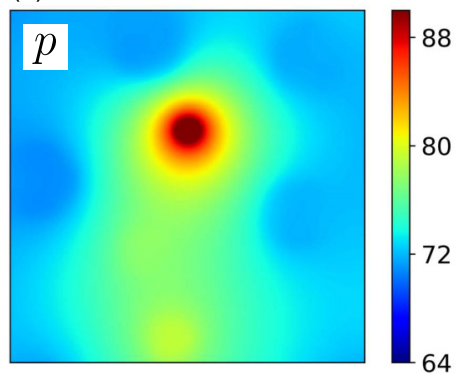

(c)
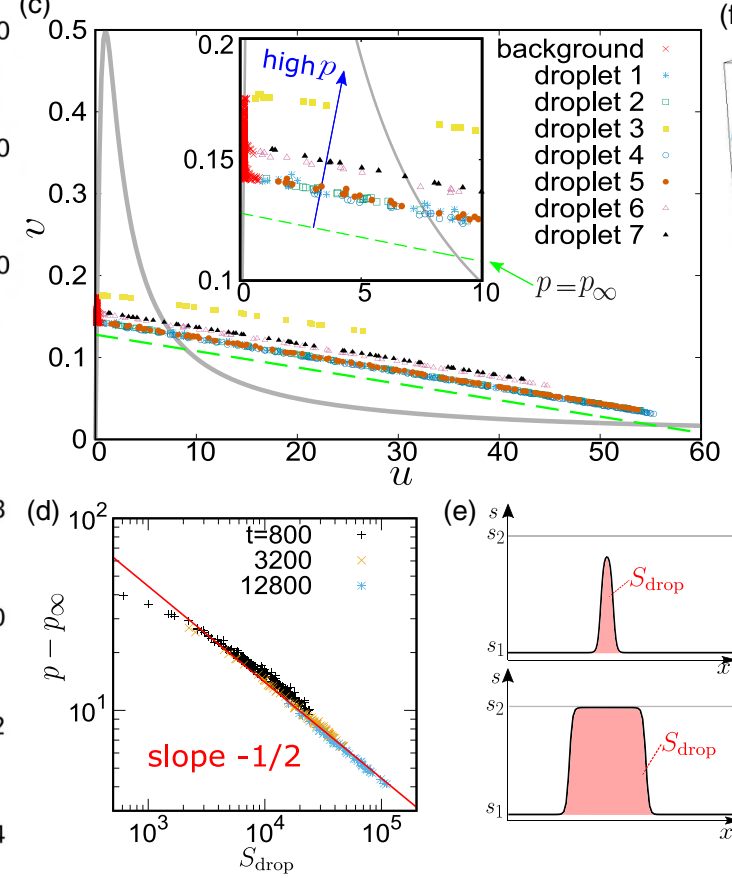

(e) $s$ (f)
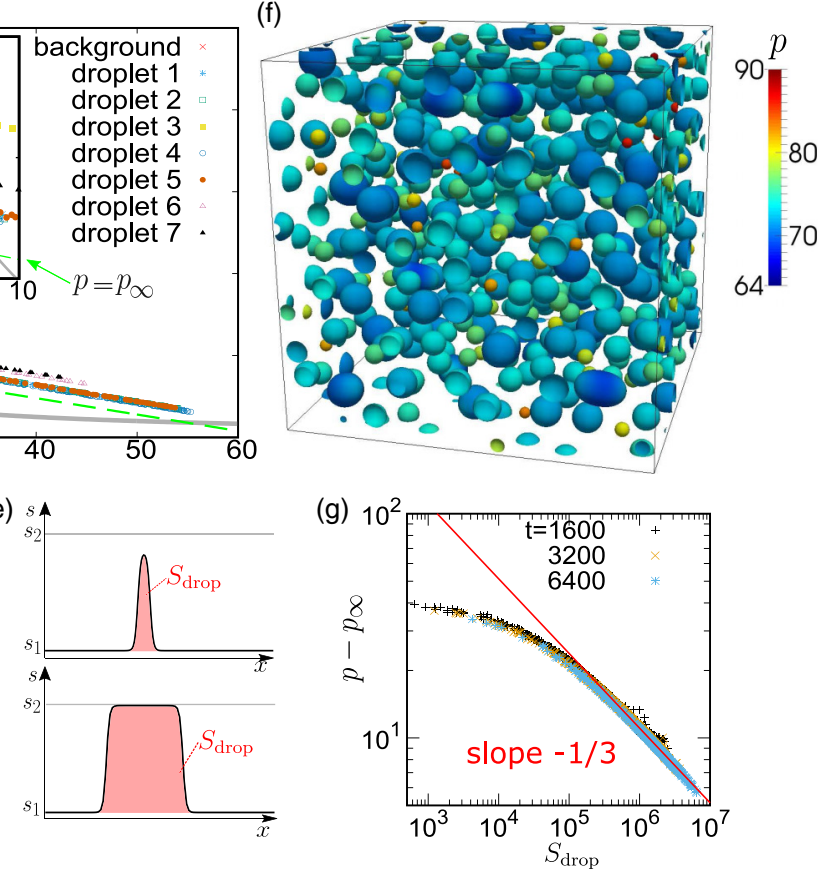

FIG. 4. Evaporation-condensation process in the coarsening regime. (a) Concentration profile of conserved variable $s$ at time $t=3200$ for parameter set A in Fig. 1(g). (b) Spatial distribution of "chemical potential" $p$ corresponding to panel (a). The image size is $256^{2}$. (c) Mapping the states of volume elements on the $u-v$ space for panels (a) and (b). Different symbols indicate to which droplets the volume elements belong [see the numbers labeled on the droplets in panel (a)]. When a volume element does not belong to any droplets, it is referred to as background. The gray curve is the nullcline of the reaction term $(f(u, v)=0)$. The green dashed line is $p_{\infty}=D_{u} u+D_{v} v$, where $p_{\infty}$ is the value of $p$ in the limit where the droplet size is sufficiently large (see below). (d) Droplet-size dependence of $p$. Mean values of $p$ for each droplet, subtracted by $p_{\infty}$, are plotted against the droplet size $S_{\text {drop }}$. Data are sampled at three different times. $p$ is expected to be equal to $p_{\infty}$ for sufficiently large droplets ( $p_{\infty} \simeq 64$ in the current parameter setting). Droplet size $S_{\mathrm{drop}}=\int\left(s-s_{1}\right) d \boldsymbol{r}$ indicates an excess amount of $s$ from the minimum stable point $s_{1}$, where the integral is taken over each droplet. The red line is a power function with exponent $-1 / d$ (where $d$ is the spatial dimension). (e) The relation between droplet size $S_{\text {drop }}$ and concentration profile $s$. For small $S_{\text {drop }}$, the maximum value of $s$ cannot reach to the largest stable point $s_{2}$ (top). For a sufficiently large $S_{\text {drop }}$, most of the body elements in the droplet have a value close to $s_{2}$ and the interface connecting the inside and outside of the droplet has an almost constant width (bottom). (f) A spatial pattern obtained from a 3D simulation for time $t=6400$ for parameter set A. A contour surface with $s=20$ is shown. The color is labeled according to the value of $p$ at the contour surface. The system size is $1024^{3}$. (g) $S_{\text {drop }}-p$ mapping obtained from 3D simulation.

conditions. The results are shown in Fig. 3(c), where the vertical axis is displayed using a logarithmic scale. The nucleation rate $I$ decreases significantly for a slight decrease in the initial concentration $u_{0}$. Such a steep change in the nucleation rate for a small change of environment is widely observed in nucleation-growth-type phase separation phenomena [45].

Although the driving force of pattern formation in the early stage is entirely different between the unstable and the metastable regions, we observe that the coarsening behavior in the late stage is very similar for these regions; small droplets evaporate while large ones grow. This similarity is quantitatively confirmed by plotting $n(t)$, the temporal change in the number density of droplets [Fig. 3(d)]. We find the same trend $\ell \propto t^{-0.30}$ as in Fig. 2(c), further supporting that the LSW mechanism can be applied to MCRDSs.

\section{Coarsening process in the late stage}

Here, we investigate the evaporation-condensation (LSW) process observed in both the unstable and metastable regions in more detail [38]. Since the time evolution of the conserved variable $s$ proceeds following the gradient of $p=D_{u} u+D_{v} v$ [see Eq. (3)], the variable $p$ is responsible for the transport of $s$ among the droplets. Figures 4(a) and 4(b) show an enlarged image of Fig. 2(a) at $t=3200$ and the corresponding spatial map of $p$, respectively. We can see that smaller droplets have a higher $p$ value. In this sense, we may call $p$ the chemical potential of $s$, on the analogy of phase separation phenomena $[22,23]$.

To verify the validity of this physical interpretation in a quantitative manner, we divide the image into $64^{2}$ equal-size square sections and map the states of the sections onto the $u-v$ space. The results are shown in Fig. 4(c). Each point in this figure corresponds to the state of each section. The symbols of the points represent the droplets to which the sections belong [see the number labeled on the droplets in Fig. 4(a)]. When a section is located outside the droplets, we use a red cross symbol (see the points labeled as background in Fig. 4(c)). We determine whether a section is inside or outside of the droplets by whether $s$ at that section is above or below a threshold value $s_{\text {th }}=2.2$, which is slightly higher than the smaller stable point $s_{1}$. In this figure, we can see that points in the same droplet are distributed on a line with constant $p$, and smaller droplets have 
higher $p$. This result indicates that the chemical-potential-like variable $p$ is uniquely determined by the morphology of the droplet, which is suggestive of the existence of the surface tension. We also confirm similar results for the 3D simulation and 2D simulation of model II [see Fig. 4(f) and Appendix E, Figs. 8 and 9, respectively].

To analyze these results in further detail, in Fig. 4(d) we show the simultaneous distribution of droplet size, $S_{\text {drop }}=$ $\int\left(s-s_{1}\right) d \boldsymbol{r}$ (the integral is taken within each droplet) and the value of $p$ in the droplets. The data are sampled at three different points in time $(t=800,3200$, and 12800). The results clearly show that there is a one-to-one correspondence between $S_{\text {drop }}$ and $p$. Furthermore, for the large $S_{\text {drop }}$ region we find the relation $\left(p-p_{\infty}\right) \propto S_{\mathrm{drop}}^{-1 / d}$ where $d$ is the spatial dimension [see Fig. 4(d)]. This asymptotic behavior can be understood as follows: when $S_{\text {drop }}$ is large, $s$ in the droplet saturates to the larger stable point $s_{2}$ and is connected to the outside of the droplet via the interface, the width of which is almost constant and independent of the droplet size [see the bottom panel of Fig 4(e)]. In this situation, $S_{\text {drop }}$ can be approximated as $S_{\text {drop }} \sim\left(s_{2}-s_{1}\right) V_{\text {drop }}$, where $V_{\text {drop }}$ is the volume (area) of the droplet. Since the droplets have a spherical shape, by denoting the radius as $R$, we can obtain the relationship $\left(p-p_{\infty}\right) \propto 1 / R$. This is the same form as the Young-Laplace equation, where the proportionality constant is expected to be associated with the surface tension in MCRDSs and will be evaluated in the subsequent section. Note that $p$ coincides with $p_{\infty}$ for a large curvature limit $(R \rightarrow \infty)$, a situation in which two stable states are segregated by a flat interface [see Sec. III A and Fig. 1(h)]. The same relationship is confirmed for the 3D simulation [see Fig. 4(g)] and a $2 \mathrm{D}$ simulation with a different reaction term [model II, see Appendix E, Fig. 9(c)]. When $S_{\text {drop }}$ is small, $s$ in the droplets is far from $s_{2}$ and the above approximation does not hold [see the top panel of Fig. 4(e)], leading to a deviation of $p-p_{\infty}$ from $\propto S_{\mathrm{drop}}^{-1 / d}$. The reason why the concentration profile of the droplets changes depending on the droplet size is that initial concentration $s_{0}$ is very far from the stable fixed point $s_{2}$; thus, it takes long time for $s$ to saturate to $s_{2}$ while retaining the mass conservation law for $s$. We note again that such a trend is not a feature specific to MCRDSs but is also seen in phase separation phenomena with a highly asymmetric shape in the free energy $[25,27-30,33,34]$.

\section{E. Evaluation of the surface tension}

As discussed above, $p-p_{\infty}$ for spherical (circular) droplets observed in coarsening regime is inversely proportional to the droplet radius $R$. Here, we derive the relationship and determine the proportionality coefficient. The governing equation for $p$ is given by

$$
\frac{\partial p}{\partial t}=\left(D_{u}+D_{v}\right) \nabla^{2} p-D_{u} D_{v} \nabla^{2} s+\left(-D_{u}+D_{v}\right) f,
$$

which, together with Eq. (3), provides a closed form of time evolution equations for a MCRDS. Consider a situation in which an isolated droplet with radius $R$ exists in infinite space under steady-state condition. The concentration profile is approximated as $s(\boldsymbol{r})=s_{\mathrm{fr}}(\zeta)$ (see Fig. 5(a)), where $s_{\mathrm{fr}}(x)=$ $\left(\left(D_{v}-D_{u}\right) u_{\mathrm{fr}}(x)+p_{\infty}\right) / D_{v}$ is the front solution discussed in
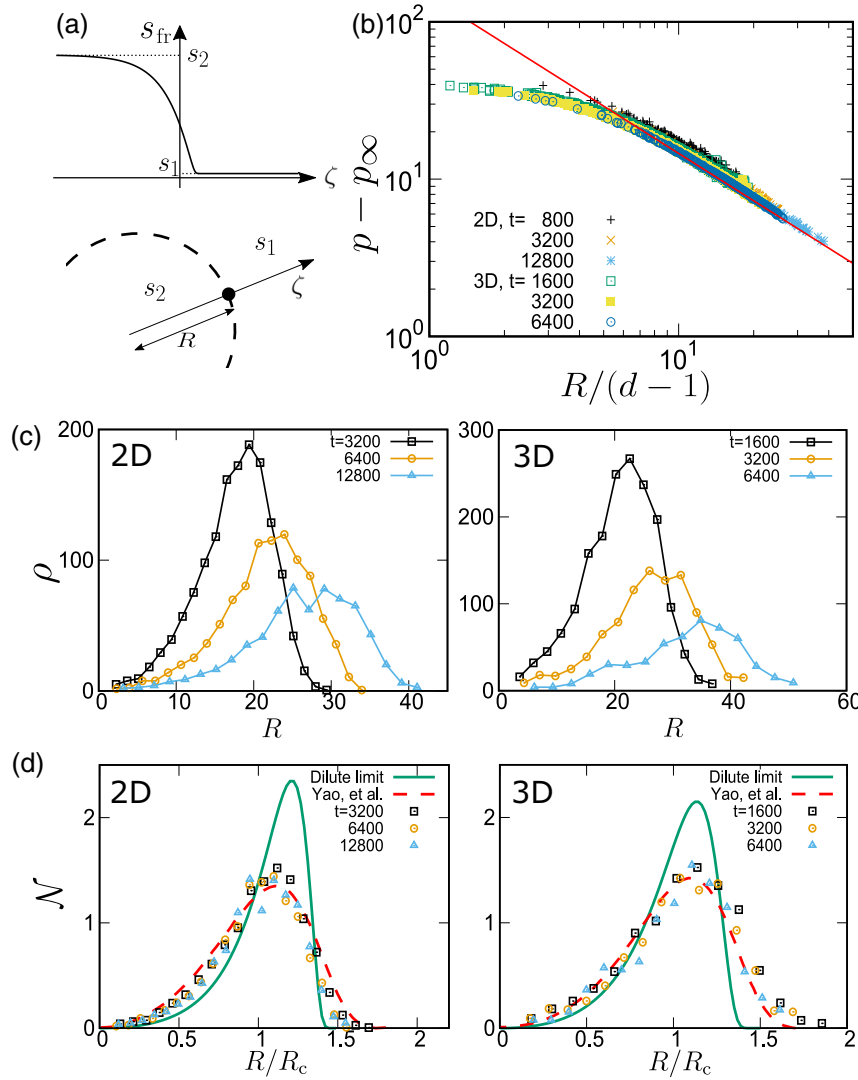

FIG. 5. Comparison with LSW theory. (a) Top: The function profile of the steady front solution $s_{\mathrm{fr}}$. Bottom: Concentration profile of $s(\boldsymbol{r})$ for a spherical droplet. Interface of a droplet is shown by the dashed line. $s(\boldsymbol{r})$ is approximated by $s_{\mathrm{fr}}(\zeta)$, where $\zeta$ is coordinate along radial direction of the droplet. (b) Relation between droplet radius $R$ and chemical-potential-like variable $p$. The data shown here is from those in Figs. 4(d) and $4(\mathrm{~g})$. Radius $R$ is evaluated using $S_{\text {tot }}=\left(s_{2}-s_{1}\right) V_{\text {drop }}$, where $V_{\text {drop }}$ is the volume of sphere with radius $R$. The red line represents theoretical relationship given by Eq. (8). (c) Droplet-size distribution $\rho(R, t)$ for 2D (left) and 3D (right) systems, respectively. (d) Dynamic scaling of $\rho$ in 2D (left) and 3D (right) systems. The symbol shows the same simulation results as in (c). The green solid and red dashed lines represent theoretical predictions for the scaled distribution $\mathcal{N}\left(R / R_{\mathrm{c}}\right)$ for a dilute volume fraction limit (the original LSW theory) and a finite volume fraction ( $\phi=8.5 \%$ and $6 \%$ for $2 \mathrm{D}$ and 3D) [26], respectively. $\mathcal{N}(x)$ is normalized to satisfy $\int \mathcal{N}(x) d x=1$. The results shown in (b)-(d) are obtained from simulations for parameter set A [see Fig. 1(g)], for which the volume fraction is $\phi=9.1 \%$.

Sec. III A. The coordinate $\zeta$ represents a radial distance of a point $\boldsymbol{r}$ from the interface of the droplet, i.e., $\zeta \equiv r-R$ where $r=|\boldsymbol{r}|$ is distance of a point $\boldsymbol{r}$ from the center of the droplet. Laplacian under $d$-dimensional spherically symmetry is written as $\nabla^{2} s=s_{\mathrm{fr}}^{\prime \prime}+[(d-1) / r] s_{\mathrm{fr}}^{\prime}$, where $s_{\mathrm{fr}}^{\prime}$ and $s_{\mathrm{fr}}^{\prime \prime}$ stand for $d s_{\mathrm{fr}} / d \zeta$ and $d^{2} s_{\mathrm{fr}} / d \zeta^{2}$, respectively. As we consider a steady state, $p$ is constant but deviates from $p_{\infty}$ owing to the curved interface [see the inset of Fig. 4(c)]. We denote the difference as $\Delta p \equiv p-p_{\infty}$. Then, insertion of the approximated steady solution $(s, p)=\left(s_{\mathrm{fr}}(\zeta), p_{\infty}+\Delta p\right)$ into Eq. (7) yields a relationship $D_{u} D_{v}(d-1) s_{\mathrm{fr}}^{\prime} / r \simeq\left(D_{v}-D_{u}\right) f_{p}\left(s_{\mathrm{fr}}, p_{\infty}\right) \Delta p$. With the expansion of the left-hand side as $1 / r=1 /(R+\zeta)=$ 
$1 / R-\zeta / R^{2}+\cdots$, and by multiplying both sides of the above relation by $s_{\mathrm{fr}}^{\prime}$ and integrating over $\zeta$, we obtain the following relationship between $\Delta p$ and $(d-1) / R$ :

$$
\Delta p \simeq \frac{D_{u} D_{v} \int s_{\mathrm{fr}}^{\prime 2} d \zeta}{\left(D_{v}-D_{u}\right) \int f_{p}\left(s_{\mathrm{fr}}, p_{\infty}\right) s_{\mathrm{fr}}^{\prime} d \zeta} \frac{(d-1)}{R},
$$

where $\Delta p$ is evaluated in the order of $O(1 / R)$ and the higher order terms are ignored. Here, we used the fact that $s_{\mathrm{fr}}^{\prime}(\zeta)$ is a localized function in the vicinity of interface $(r=R)$ with exponential decay and thus integration range is extended to $-\infty<\zeta<\infty$.

Equation (8) is a relationship analogous to the YoungLaplace's law [22,23], in which the coefficient defines "surface tension" $\gamma$. Note that this definition does not rely on variational structure; although the concept of effective surface tension is well established in some RDSs such as onecomponent bistable RDSs where the governing equations can be rewritten into variational form (see, e.g., Refs. [51-53]), the existence of variational structure in MCRDSs is under debate [13,31]. Using the front solution $s_{\mathrm{fr}}(x)$ [see Fig. 1(d)], we calculate $\gamma \simeq 146$. In Fig. 5(b), we replot $p-p_{\infty}(=\Delta p)$ in Figs. 4(d) and 4(e) against $R /(d-1)$, where $R$ is evaluated from the volume (area) of the droplet $V_{\text {drop }}$. All the data in different times and dimensions are mapped onto a single master curve, which asymptotically obeys Eq. (8) for large $R$ [see the red curve Fig. 5(b)]. The similar results are confirmed for model II [see Appendix E and Fig. 9(d)]. These results further indicate that surface-tension-like quantity is relevant to the coarsening process in MCRDSs.

\section{F. Droplet-size distribution by LSW analysis}

The distribution of droplet size is an important universal characteristics of coarsening processes that can be rather easily observed. Figure 5(c) shows droplet-size distribution in $2 \mathrm{D}$ and $3 \mathrm{D}$ systems at various times. Using the surfacetension-like quantity $\gamma$ obtained above, here we evaluate the droplet-size distribution in MCRDSs by employing the LSW theory (a mean-field theory for a droplet-size distribution) $[23,24,26]$.

First, let us consider the growth (or shrinkage) rate of a droplet with radius $R(t)$. Due to mass conservation, the time variation in $R$ occurs only through diffusion as represented by Eq. (3). By substituting the concentration profile of a droplet $s(\boldsymbol{r})=s_{\mathrm{fr}}(r-R(t))$ into Eq. (3) and taking an integral along radial coordinate across the interface, we obtain an equation $\left(s_{2}-s_{1}\right) d R / d t \simeq p_{r}(R-\epsilon)-p_{r}(R+\epsilon)$, where $p_{r} \equiv \partial p / \partial r$ and $\epsilon$ is a value sufficiently larger than the interfacial width. Spatial profile of $p$ is evaluated as follows; because spatial dependence of $p$ is small [in the order of $O(1 / R)$ ], the time evolution of $R(t)$ is sufficiently slow and the time dependence of $p$ is subject to $R$. Hence, $p$ obeys the Poisson equation $\nabla^{2} p=0$ for given value of $R(t)$. At $d=3$, the compatible solution with Eq. (8) is

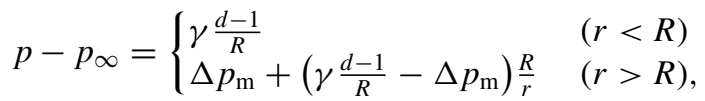

where $p_{\infty}+\Delta p_{\mathrm{m}}$ is a mean-field value of $p$ in the background region, which is determined by the distribution of droplets. By putting the above arguments together, the growth rate of a droplet is derived as

$$
j \equiv \frac{d R}{d t}=\frac{\alpha}{R}\left(\frac{1}{R_{\mathrm{c}}}-\frac{1}{R}\right),
$$

where $\alpha=2 \gamma /\left(s_{2}-s_{1}\right)$ and $R_{\mathrm{c}}=\Delta p_{\mathrm{m}} / 2 \gamma . R_{c}$ represents a critical radius; droplets with radius larger than $R_{c}$ will grow, while those with smaller radius will shrink. For 2D systems, by logarithmic correction of time, the same form of equation as Eq. (9) is derived $[23,24,26]$.

Then, we consider the droplet-size distribution $\rho(R, t)$, where $\rho(R, t) d R$ represents the number of droplet per unit volume with radius between $R$ and $R+d R$ at time $t$. Time evolution of $\rho(R, t)$ is governed by a continuity equation $\partial_{t} \rho+\partial_{R}(j \rho)=0$, where $j$ is provided by Eq. (9). Note that because $\Delta p_{\mathrm{m}}$ depends on the droplet distribution, $\rho(R, t)$ must be solved in a self-consistent manner. By assuming that the droplet-size distribution is self-similar, i.e., $\rho(R, t)$ is in the form of $\rho(R, t) \propto \mathcal{N}\left(R / R_{\mathrm{c}}(t)\right)$, the LSW theory predicts that the critical radius is

$$
R_{\mathrm{c}}(t)=(c \alpha t)^{1 / 3},
$$

as well as the function form of $\mathcal{N}(x)[23,24,26]$. In the original LSW theory, the volume fraction of the minority phase $\phi$ was assumed to be infinitesimally small (dilute limit), whereby $c$ was estimated as $c=4 / 9$ for a $3 \mathrm{D}$ system. Below, we will compare our data in Fig. 5(c) with the LSW theory of zeroand finite-volume fraction; for the latter, we refer to Yao et al. [26].

In our MCRDS (model I at parameter set A), the volume fraction is $\phi=\left(s_{2}-s_{0}\right) /\left(s_{2}-s_{1}\right) \simeq 9.1 \%$. $c$ at this volume fraction is approximately 0.6 and 0.9 for $2 \mathrm{D}$ and $3 \mathrm{D}$ cases, respectively, while $\alpha$ is given by $\alpha=(d-1) \gamma /\left(s_{2}-s_{1}\right)$ [26]. Equation (10) predicts $R_{\mathrm{c}}=17.3,21.9$, and 27.5 at time $t=$ 3200,6400 , and 12800 , respectively, for $2 \mathrm{D}$. For $3 \mathrm{D}, R_{\mathrm{c}}=$ $19.9,25.0$, and 31.5 at $t=1600,3200$, and 6400 , respectively. These values agree with the mean value of the distribution obtained from our simulations [see Fig. 5(c)]. Further, as shown in Fig. 5(d), in each 2D and 3D system, the normalized distributions of radius scaled by $R_{\mathrm{c}}$ at different times collapse to a single curve, which agrees well with a theoretical prediction for finite volume fraction (see the red dashed line; we also show those estimated for $\phi \rightarrow 0$ by the green solid line). Here, we used the results by Yao et al. [26] at $\phi=8.5 \%$ for $2 \mathrm{D}$ and $6.0 \%$ for $3 \mathrm{D}$. Note that the function form of $\mathcal{N}(x)$ almost unchanged above these volume fractions [24]. In summary, our data support that the evaporation-condensation process in MCRDSs well follows the LSW theory under our definition of surface tension Eq. (8).

\section{CONCLUSION}

In conclusion, we found that the pattern dynamics in MCRDSs, starting from a uniform state toward an eventual single isolated domain, are classified into two types, similar to phase separation phenomena (see also Ref. [13]). One is triggered by the instability of the initial uniform state and the other goes through a nucleation and growth process. This classification is systematically addressed via a phase diagram, which can be constructed by the instability condition [Eq. (4)] and the relation for the bistable states [Eq. (5)]. 
We performed large-scale numerical simulations in both two and three dimensions. We confirmed that in the late stage, droplet patterns appearing both in stable and metastable regions obey a relation similar to the Young-Laplace equation [Eq. (8)] and coarsen with the same growth exponent as in the evaporation-condensation (or LSW) mechanism. Furthermore, we derived a Young-Laplace-like relation from the MCRD equations and defined the proportionality coefficient as surface tension. Based on this quantity, we extended the LSW theory to MCRDSs and found that the droplet-size distribution obtained from simulations shows good agreement with the theoretical prediction. These results suggest that a surface-tension-like quantity is relevant to MCRDSs and plays a key role in the coarsening dynamics, which provides insight into molecular self-assembly driven by chemical reactions.

In the present paper, we derived "surface tension" from a Young-Laplace-like relation [Eq. (8)] and found it is relevant to the coarsening. While the coarsening behaviors in MCRDSs are similar to those in classical phase separation systems which have variational form, in general, phase separation systems including nonvariational terms tend to show suppression of the coarsening [54,55]. Thus, we expect that the physical origin of the surface tension in MCRDSs could be further clarified through possible variational structures, as in one-component nonequilibrium systems such as autocatalytic [51-53] or self-propelled particles [56,57]. In this context, it is notable that Chan-Hilliard-like equations have recently been derived from MCRDSs via a weakly nonlinear analysis by focusing on bifurcation points [13,31], and that violation of mass conservation in RDSs can suppress coarsening processes $[12,14]$. This knowledge could contribute further to our understanding of MCRDSs, including its energetic description based on variational structures.

MCRDSs were originally introduced and have been discussed as models for molecular localization such as membrane-bounded GTPases which are responsible for the formation of cell polarity [5-14] and neuron dendrites [58] and phosphoinositide lipids [59]. Recently, MCRDSs have been also applied to various systems such as oscillatory motion in Min-protein [35] and chemical turbulence [36], by which the importance of MCRDSs for pattern formation in biological systems is further recognized. Although the details of chemical reactions are significantly different among the systems, MCRDSs show universal dynamics and can be relevant for many existing systems. One interesting possible application of MCRDSs is for liquid-liquid phase separation observed inside cells [60-63]. Liquid droplets found in cells contain many chemical components that regulate each other; thus, some droplets may be formed by chemical reactions consuming cellular energy rather than nonmolecular specific physical interactions among proteins. MCRDSs can provide a natural framework to model and understand the general mechanism of such phenomena.

\section{ACKNOWLEDGMENTS}

Numerical simulations were performed on the SGI ICE XA/UV hybrid system at the ISSP at the University of Tokyo. This study was supported by JSPS KAKENHI (JP20K14424 and JP18H01185) and JST CREST (JPMJCR1923).

\section{APPENDIX A: MCRDS WITH FLUCTUATIONS}

The mass-conserved reaction-diffusion equation including fluctuations is given as follows:

$$
\begin{aligned}
& \partial_{t} u=D_{u} \nabla^{2} u-f(u, v)+\nabla \cdot \boldsymbol{j}_{u}-\eta_{r}, \\
& \partial_{t} v=D_{v} \nabla^{2} v+f(u, v)+\nabla \cdot \boldsymbol{j}_{v}+\eta_{r} .
\end{aligned}
$$

Here $D_{u}$ and $D_{v}$ are the diffusion coefficients of chemical species, $U$ and $V$, and we assume $D_{u}<D_{v} . f$ is the reaction term. $\boldsymbol{j}_{u}$ and $\boldsymbol{j}_{v}$ are fluctuations accompanying with diffusion of $U$ and $V$, respectively, satisfying the below statistics:

$$
\begin{aligned}
& \left\langle j_{u, \alpha}(t, \boldsymbol{r}) j_{u, \beta}\left(t^{\prime}, \boldsymbol{r}^{\prime}\right)\right\rangle=2 D_{u} u \delta\left(t-t^{\prime}\right) \delta\left(\boldsymbol{r}-\boldsymbol{r}^{\prime}\right) \delta_{\alpha, \beta}, \\
& \left\langle j_{v, \alpha}(t, \boldsymbol{r}) j_{v, \beta}\left(t^{\prime}, \boldsymbol{r}^{\prime}\right)\right\rangle=2 D_{v} v \delta\left(t-t^{\prime}\right) \delta\left(\boldsymbol{r}-\boldsymbol{r}^{\prime}\right) \delta_{\alpha, \beta} .
\end{aligned}
$$

$\eta_{r}$ is fluctuation accompanying with reaction and satisfies

$$
\left\langle\eta_{r}(t, \boldsymbol{r}) \eta_{r}\left(t^{\prime}, \boldsymbol{r}^{\prime}\right)\right\rangle=\sum_{i}\left|\operatorname{Reaction}_{i}\right| \delta\left(t-t^{\prime}\right) \delta\left(\boldsymbol{r}-\boldsymbol{r}^{\prime}\right) .
$$

In our study, we use a reaction term of the form $f=f_{+}-f_{-}$ where $f_{+}=k_{u} u$ and $f_{-}=k_{v} v$. In this case, $\sum_{i}\left|\operatorname{Reaction}_{i}\right|=$ $f_{+}+f_{-}$.

\section{APPENDIX B: NONDIMENSIONALIZATION}

By denoting the time and space units as $\tau$ and $\ell$, respectively, and taking the units for $u, v$ and $f$ as $u_{0}, v_{0}$ and $f_{0}$, respectively, we can rewrite Eqs. (A1) and (A2) as

$$
\begin{gathered}
\frac{\partial}{\partial \tilde{t}} \tilde{u}=\tilde{\nabla}^{2} \tilde{u}-\mathcal{R} \tilde{f}+\sqrt{\mathcal{R}} \sigma \tilde{\nabla} \cdot \tilde{\boldsymbol{j}}_{u}-\mathcal{R} \sigma \tilde{\eta}_{r}, \\
\frac{\partial}{\partial \tilde{t}} \tilde{v}=\mathcal{D} \tilde{\nabla}^{2} \tilde{v}+\tilde{f}+\sqrt{\mathcal{D}} \sigma \tilde{\nabla} \cdot \tilde{\boldsymbol{j}}_{v}+\sigma \tilde{\eta}_{r},
\end{gathered}
$$

where we define $\mathcal{R}=v_{0} / u_{0}, \mathcal{D}=D_{v} / D_{u}, \sigma=1 / \sqrt{v_{0} \ell^{d}}$, $\ell=\sqrt{D_{u} \tau}$, and $\tau=v_{0} / f_{0} . \quad \tilde{A}$ represents scaled variable of $A$. The statistics of the noise terms are then given as $\left\langle\tilde{j}_{u, \alpha}(\tilde{t}, \tilde{\boldsymbol{r}}) \tilde{j}_{u, \beta}\left(\tilde{t}^{\prime}, \tilde{\boldsymbol{r}}^{\prime}\right)\right\rangle=2 \tilde{u} \delta\left(\tilde{t}-\tilde{t}^{\prime}\right) \delta\left(\boldsymbol{r}-\boldsymbol{r}^{\prime}\right) \delta_{\alpha, \beta}$, $\left\langle\tilde{j}_{v, \alpha}(\tilde{t}, \tilde{\boldsymbol{r}}) \tilde{j}_{v, \beta}\left(\tilde{t}^{\prime}, \tilde{\boldsymbol{r}}^{\prime}\right)\right\rangle=2 \tilde{v} \delta\left(\tilde{t}-\tilde{t}^{\prime}\right) \delta\left(\tilde{\boldsymbol{r}}-\tilde{\boldsymbol{r}}^{\prime}\right) \delta_{\alpha, \beta} \quad$ and $\left\langle\tilde{\eta}_{r}(\tilde{t}, \tilde{\boldsymbol{r}}) \tilde{\eta}_{r}\left(\tilde{t}^{\prime}, \tilde{\boldsymbol{r}}^{\prime}\right)\right\rangle=\left(\tilde{f}_{+}+\tilde{f}_{-}\right) \delta\left(\tilde{t}-\tilde{t}^{\prime}\right) \delta\left(\tilde{\boldsymbol{r}}-\tilde{\boldsymbol{r}}^{\prime}\right) \delta_{\alpha, \beta}$.

We chose $u_{0}$ and $v_{0}$ independently such that the scaled reaction term $\tilde{f}(\tilde{u}, \tilde{v})$ becomes parameter-free, which has advantages in constructing phase diagram; the condition for type-II ${ }_{s}$ instability Eq. (4) is scaled as $\tilde{f}_{\tilde{u}}-\tilde{f}_{\tilde{v}} / \mathcal{R} \mathcal{D}<0$. On the other side, bistable points in the steady-state solution, $\left(\tilde{u}_{1}\right.$, $\left.\tilde{v}_{1}\right)$ and $\left(\tilde{u}_{2}, \tilde{v}_{2}\right)$, are obtained using two equalities $\tilde{f}(\tilde{u}, \tilde{v})=0$ and $\tilde{u}+\mathcal{R} \mathcal{D} \tilde{v}=\tilde{p}_{\infty}$ [see Fig. 1(c)], where we use $D_{u} u_{0}$ as the unit for $p$. Because $\tilde{f}$ is parameter-free, the existence of such a solution depends solely on $\mathcal{R D} \equiv 1 / T$ as shown in Fig. 1(g). For model I $\left(k_{u}=c a /\left(b+u^{2}\right), k_{v}=c\right)$, we set $u_{0}=$ $\sqrt{b}, v_{0}=a / \sqrt{b}$ and $f_{0}=a c / \sqrt{b}$, and the resulting reaction term is $\tilde{f}=\tilde{u} /\left(1+\tilde{u}^{2}\right)-\tilde{v}$. For model II $\left(k_{u}=\alpha, k_{v}=k_{0}+\right.$ $\left.\beta u^{2} /\left(K^{2}+u^{2}\right)\right)$, we set $u_{0}=K, v_{0}=\alpha K / \beta, f_{0}=\alpha K$, and $k_{0}=0$, and the resulting reaction term is $\tilde{f}=\tilde{u}-\tilde{u}^{2} \tilde{v} /\left(1+\tilde{u}^{2}\right)$. For model I, $T<1 / 8\left(\equiv T_{\mathrm{c}}\right.$ ) is necessary for the condition for type- $\mathrm{II}_{s}$ instability and $T_{\mathrm{c}}$ is the value of $T$ at the vertex of a parabolic function shown in Fig. 1(g). Such an upper bound does not exist for model II [see Fig. 7(b)].

From Eqs. (B1) and (B2), we can immediately obtain the time evolution equation for the conserved variable $\tilde{s}=\tilde{u}+R \tilde{v}$ 

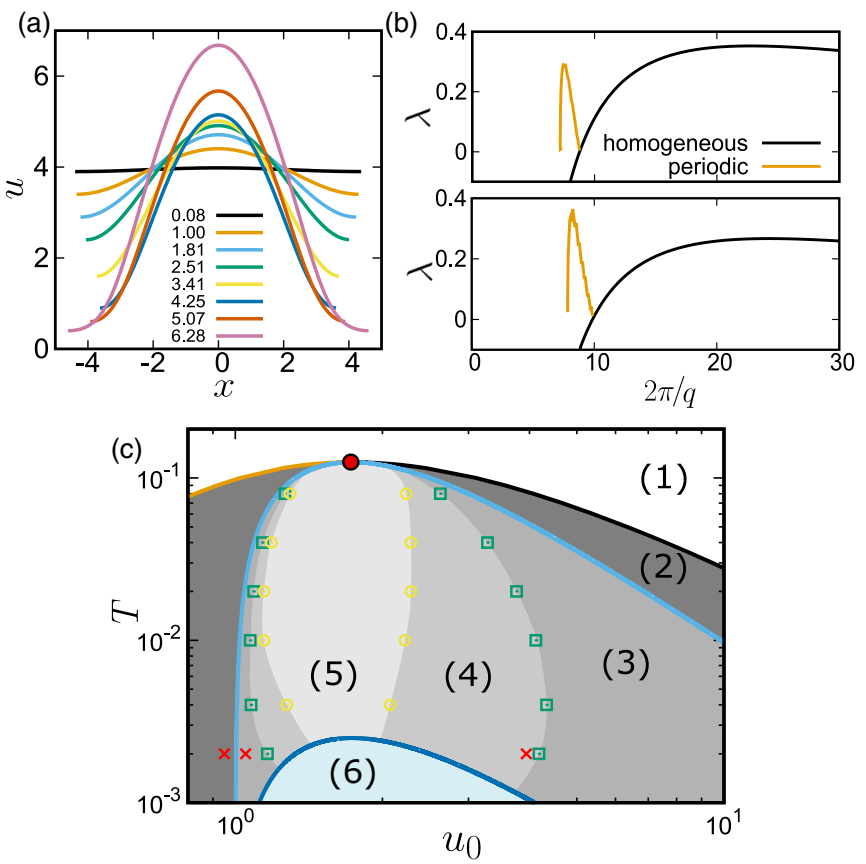

FIG. 6. Linear stability of steady periodic solutions. (a) Periodic solutions with fixed conserved quantity. The label indicates the amplitude of each solution [i.e., difference between the maximum and minimum of $u(x)]$ at $\left(u_{0}, T\right)=(3.94,0.002)$. (b) Comparison of the growth rate between homogeneous (black) and periodic (brown) solutions at $\left(u_{0}, T\right)=(3.94,0.002)$ and $(4.5,0.002)$ (top and bottom panels, respectively). The horizontal axis represents the period of solutions. (c) Phase diagram where the linear stability of periodic solutions is taken into consideration. Regions (1) and (2) are equivalent to the unstable and metastable regions in Fig. 1(g). (3) and (4) indicate the regions where the maximum growth rate for homogeneous solutions is larger and less than that for periodic ones, respectively. In region (5), no periodic solution has positive growth rate. The green squares and yellow circles represent the borders between regions (3) and (4), and (4) and (5), respectively, which are determined numerically. Region (6) indicates parameter set in which the homogeneous solution is unstable (see the second paragraph of Sec. III A for details). See Fig. 1(g) for the definitions of the other symbols.
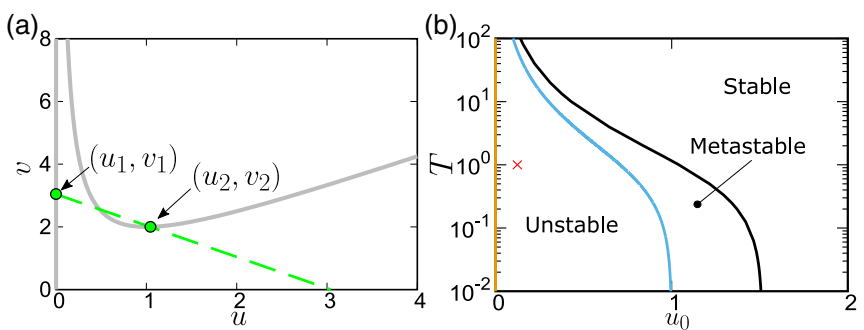

FIG. 7. Steady-state analysis for model II. (a) Mapping of the steady stable points on the $u-v$ space for $T=1$. The gray curve is the nullcline for the reaction term $f$. The green dashed line represents $p_{\infty}=D_{u} u+D_{v} v$. The green circles indicate the stable fixed points. (b) Phase diagram. The blue curve represents the border of the unstable region where type- $\mathrm{II}_{\mathrm{s}}$ instability is operative. The black and gray curves are the set composed of the steady stable points, $u_{1}$ and $u_{2}$, respectively [see the green circles in (a)]. Here we focus on the pattern formation process at the red cross point $\left(u_{0}=0.125, T=1\right)$. as

$$
\tilde{\partial}_{t} \tilde{s}=\tilde{\nabla}^{2} \tilde{p}+\sqrt{\mathcal{R}} \sigma \tilde{\nabla} \cdot \tilde{\boldsymbol{j}}_{p},
$$

where $\tilde{p}=\tilde{u}+\mathcal{R} \mathcal{D} \tilde{v}$. Here we use $u_{0}$ and $D_{u} u_{0}$ as units for $s$ and $p$, respectively. The last term on the right-hand side represents the diffusion noise that satisfies $\left\langle\tilde{j}_{p, \alpha}(\tilde{t}, \tilde{\boldsymbol{r}}) \tilde{j}_{p, \beta}\left(\tilde{t}^{\prime}, \tilde{\boldsymbol{r}}^{\prime}\right)\right\rangle=$ $2 \tilde{p} \delta\left(\tilde{t}-\tilde{t}^{\prime}\right) \delta\left(\tilde{\boldsymbol{r}}-\tilde{\boldsymbol{r}}^{\prime}\right) \delta_{\alpha, \beta}$.

All the data presented in the figures are normalized by the units introduced here.

\section{APPENDIX C: DERIVATION OF EQ. (5)}

We consider a solution for MCRDSs in one dimension, where $(u, v)$ approaches $\left(u_{1}, v_{1}\right)$ and $\left(u_{2}, v_{2}\right)$ for $x \rightarrow-\infty$ and $+\infty$, respectively. In a steady condition, Eq. (3) becomes $\nabla^{2} p=0$ and the general solution is given by $p=A x+B$ where $A$ and $B$ are constants. If $A$ is nonzero, $p$ (and thus, at least one of $u$ or $v$ ) diverges for $x \rightarrow \pm \infty$, which is unphysical. Thus, $p$ has a constant value, which we denote as $p_{\infty}$. As discussed in the main text, we consider a solution that propagates with a constant velocity $c$ [32]. Substituting $u(x, t)=u(x-c t)$ into Eq. (1), we obtain the following relationship:

$$
-c \frac{d u}{d z}=D_{u} \frac{d^{2} u}{d z^{2}}-f\left(u, \frac{p_{\infty}-D_{u} u}{D_{v}}\right),
$$

where $z=x-c t$ is a moving coordinate at speed $c$. By multiplying the above equation by $d u / d z$ and taking integral over the entire space, we obtain

$$
\begin{array}{r}
-c \int_{-\infty}^{\infty}\left(\frac{d u}{d z}\right)^{2} d z=\int_{u_{1}}^{u_{2}} \frac{D_{u}}{2} \frac{d}{d z}\left(\frac{d u}{d z}\right)^{2} d u \\
-\int_{u_{1}}^{u_{2}} f\left(u, \frac{p_{\infty}-D_{u} u}{D_{v}}\right) d u .
\end{array}
$$

The first term on the right-hand side vanishes because $d u / d z=0$ for $z= \pm \infty$. Therefore, $c$ is related to $u(z)$ as follows:

$$
c=\left[\Psi\left(u_{2}, p_{\infty}\right)-\Psi\left(u_{1}, p_{\infty}\right)\right] / \int_{-\infty}^{\infty}\left(\frac{d u}{d z}\right)^{2} d z .
$$

Here, $\Psi$ is an integral of $f$ with respect to $u$ introduced in Eq. (6). At the stationary state where $c=0$, two stable states are related by $\Psi\left(u_{2}, p_{\infty}\right)=\Psi\left(u_{1}, p_{\infty}\right)$. This equality determines a position-independent value of $p=p_{\infty}$. Note also that $u_{1}$ and $u_{2}$ are two minima of $\Psi\left(u, p_{\infty}\right)$, satisfying $d \Psi\left(u, p_{\infty}\right) / d u=f\left(u,\left(p_{\infty}-D_{u} u\right) / D_{v}\right)=0$. In the main text, $p_{\infty}$ is evaluated by assuming the periodic boundary conditions for which the system size $L$ is sufficiently large.

The existence of the static stationary solution can be confirmed by introducing a functional defined as $\mathscr{E}[u, p] \equiv$ $\int d \boldsymbol{r}\left[\Psi(u, p)+\frac{D_{u}}{2}|\nabla u|^{2}\right]$. The functional derivative of $\mathscr{E}$ with respect to $u$ relates to the time derivative of $u$ as $\partial u / \partial t=$ $-\delta \mathscr{E} / \delta u$. Under the condition of constant $p=p_{\infty}, \mathscr{E}\left[u, p_{\infty}\right]$ acts as a Lyapunov function because

$$
\frac{d \mathscr{E}\left[u, p_{\infty}\right]}{d t}=-\int\left(\frac{\partial u}{\partial t}\right)^{2} d \boldsymbol{r} \leqslant 0 .
$$

If we assume a traveling solution of the form $u(x-c t)$, $d \mathscr{E}\left[u, p_{\infty}\right] / d t=-c^{2} \int(d u / d z)^{2} d z$ and $c=0$ is the only 
compatible solution at the stationary state. It is worth mentioning that when reaction rates are in the form of $k_{u}=k_{u}(u)$ and $k_{v}=$ const as in model I, $\mathscr{E}$ works as Lyapunov function commonly for $u$ and $p$ [64].

\section{APPENDIX D: LINEAR STABILITY ANALYSIS OF STEADY PERIODIC SOLUTIONS}

We evaluate the linear stability of periodic steady solutions in MCRDSs, which is known to modulate the short-time evolution of patterns in spinodal-type phase separation $[33,34,37]$. First, for a given parameter set of $\left(u_{0}, T\right)$, we numerically search a family of periodic steady solutions $\left(u^{*}(x), p^{*}\right)$. Examples of the periodic solutions at $\left(u_{0}, T\right)=$ $(3.94,0.002)$ [parameter set A, see Fig. $1(\mathrm{~g})$ ] are shown in Fig. 6(a). These solutions have the same conserved quantity $s_{0}$ but different spatial periods. Next, we evaluate the linear stability of these solutions. By denoting $(u, p)=\left(u^{*}(x)+\right.$ $\left.\delta u(x), p^{*}+\delta p(x)\right)$, a linear equation with respect to $\boldsymbol{w} \equiv$ $(\delta u, \delta p)^{T}$ is obtained as $\partial_{t} \boldsymbol{w}=\mathcal{L} \boldsymbol{w}$, where $\mathcal{L}$ is a linear operator given by

$$
\mathcal{L}=\left(\begin{array}{cc}
D_{u} \nabla^{2}-f_{u} & -f_{p} \\
\left(D_{v}-D_{u}\right)\left(-D_{u} \nabla^{2}+f_{u}\right) & D_{v} \nabla^{2}+\left(D_{v}-D_{u}\right) f_{p}
\end{array}\right) .
$$

Here, $f_{u}$ and $f_{p}$ represent $\partial f(u, p) / \partial u$ and $\partial f(u, p) / \partial p$ at $(u, p)=\left(u^{*}, p^{*}\right)$, respectively. The maximum eigenspectrums of $\mathcal{L}$ obtained for various $\left(u^{*}, p^{*}\right)$ yield the growth rate of the periodic solutions, which is a quantity characterizing the stability of the solutions. Figure 6(b) (brown lines) shows the linear growth rate plotted against the period of the solutions at parameter set $\left(u_{0}, T\right)=(3.94,0.002)$ and $(4.5,0.002)$. In this figure, the growth rates for homogeneous solution are also shown, which are obtained simply by plotting $\lambda$ with respect to $2 \pi / q$ in Fig. 1(b). The positive growth rate of the periodic solution indicates that finite perturbation to the initial homogeneous state can cause amplification of unstable modes that are missing in the linear stability analysis at the homogeneous state.

(a)
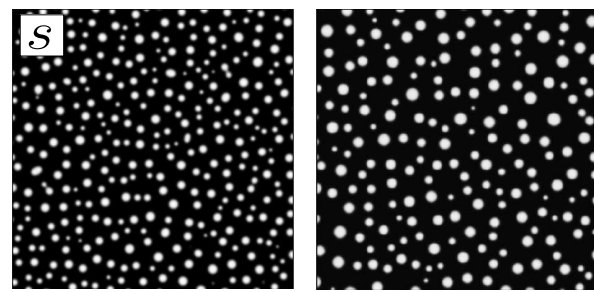

(b)

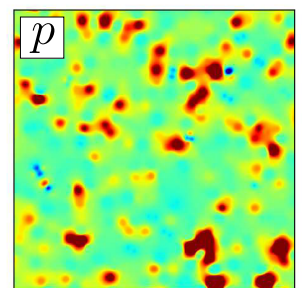

$t=2.0 \times 10^{4}$

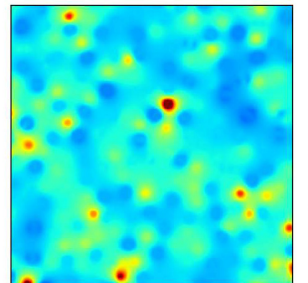

$7.0 \times 10^{5}$
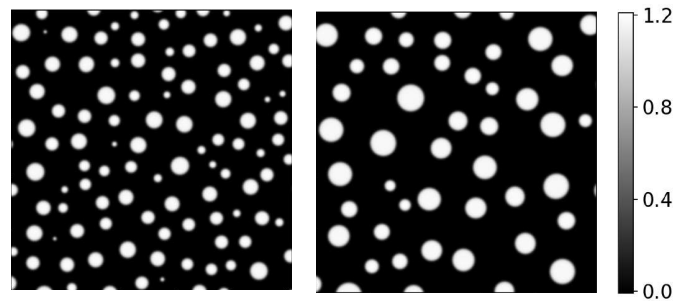

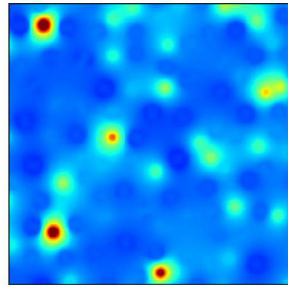

$3.0 \times 10^{5}$

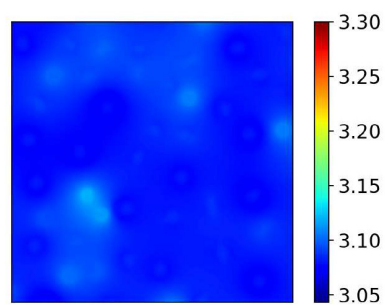

$1.2 \times 10^{6}$

FIG. 8. Temporal change of the pattern at $\left(u_{0}, T\right)=(0.125,1)$. (a) and (b) show the temporal changes in the field variables $s$ and $p$, respectively. The side length of the images is 10240 . The minimum value of the color bar for $p$ is set to $p=p_{\infty} \sim 3.04$. 

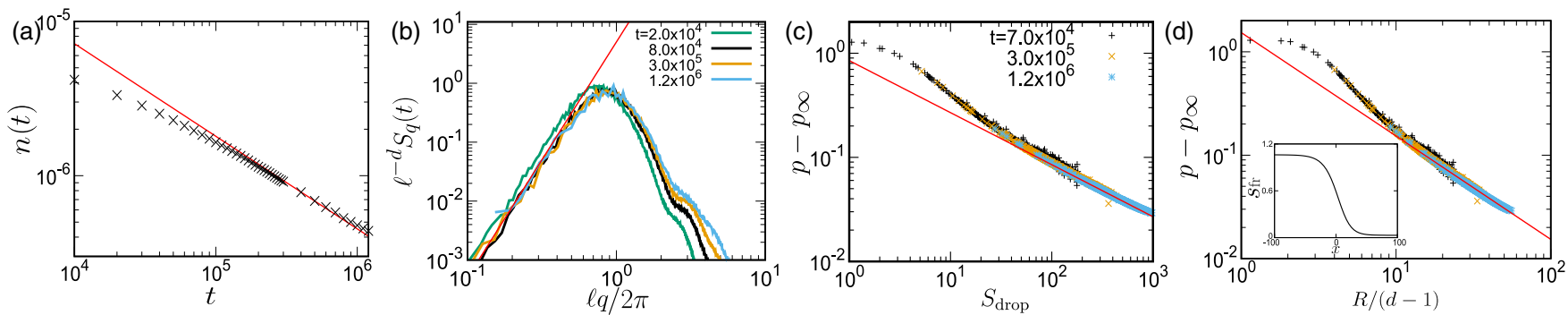

FIG. 9. Analysis of the coarsening process at $\left(u_{0}, T\right)=(0.125,1)$. (a) Temporal changes of the number of droplets per unit volume $n(t)$. The red line represents a power-law function with a slope of 0.60. (b) Scaled structure factor $\ell^{-d} S(q, t)$ (where $d$ is the spatial dimension) at various time. $\ell(t)$ is a length scale determined by $\ell^{d} n=1$, which approximately corresponds to a typical distance between neighboring droplets. The red line represents a power function with a slope of 4. (c) Droplet size $\left(S_{\text {drop }}\right)$ dependence of chemical-potential-like variable $p$. The red line is a power function with an exponent of $-1 / d$ ( $d$ being the spatial dimension; $d=2$ in the current case). (d) $p-p_{\infty}$ as a function of $R /(d-1)$. Here, droplet radius $R$ is obtained from $S_{\text {drop }}$ in the same manner as in Fig. 5(b). The red line represents $p-p_{\infty}=\gamma(d-1) / R$. The coefficient $\gamma=1.53$ is calculated through Eq. (8) using the steady front solution $s_{\mathrm{fr}}$ (see the inset) as the input.

and drawing the full phase diagram for model II requires an additional axis representing $k_{0}$.

Below we present the results of coarsening dynamics in $2 \mathrm{D}$ at point $\left(u_{0}, T\right)=(0.125,1)$ [see the red cross symbol in Fig. 7(b)]. Figure 8(a) shows the temporal change of pattern (the concentration field of $s$ ), where dropletlike patterns form in the early time $\left(t=2.0 \times 10^{4}\right)$ and coarsen over time. Figure 9(a) shows the temporal change of the number density of the droplets $n(t)$, which asymptotically approaches a powerlaw function with exponent -0.60 (red line). Thus, the growth rate is $0.60 / d=0.30(d=2)$, which is coincident with the results for model I [Figs. 2(c) and 3(d)].

Figure 9(b) shows the scaled structure factor, where we can see that the structure factors at different times are scaled for both small and large $q$ after the system reaches the coarsening regime where $n(t)$ shows a power-law decay. In Fig. 2(d), we have seen that the scalability for large $q$ in intermediate time regime does not hold for model I. This difference is ascribed to whether the concentration inside the droplets is saturated to the stable fixed point $s_{2}$ [see Fig. 1(a)]. For the current parameter choice for model II, the values of the bistable steady solution $s_{1}$ and $s_{2}$ are not so far from that of the initial homogeneous state. The concentration inside the droplets easily saturates to the stable points, and as a result, the system shows a self-similar coarsening.

Figure 8(b) shows the map of $p$ at the same spatial region and time as Fig. 8(a), where we can see a trend similar to Fig. 4(b). This similarity, together with the growth exponent 0.30 , indicates that the evaporation-condensation mechanism also works in model II. In Fig. 9(c), a power-law relationship between $p-p_{\infty}$ and the droplet size with exponent $-1 / d$ is confirmed as in model I [see Fig. 4(d) and 4(g)]. Moreover, as shown in Fig. 9(d), the relationship Eq. (8) between droplet radius $R$ and $p-p_{\infty}$ is also confirmed. These results indicate that a surface-tension-like quantity generally exists in MCRDSs without depending on details of the reaction terms, and plays a crucial role in the coarsening process of the patterns.
[1] M. C. Cross and P. C. Hohenberg, Rev. Mod. Phys. 65, 851 (1993).

[2] P. C. Bressloff, J. D. Cowan, M. Golubitsky, P. J. Thomas, and M. C. Wiener, Neural Comput. 14, 473 (2002).

[3] R. Hoyle, Pattern Formation: An Introduction to Methods (Cambridge University Press, Cambridge, 2006).

[4] E. Meron, Math. Biosci. 271, 1 (2016).

[5] M. Otsuji, S. Ishihara, C. Co, K. Kaibuchi, A. Mochizuki, and S. Kuroda, PLOS Comput. Biol. 3, e108 (2007).

[6] S. Ishihara, M. Otsuji, and A. Mochizuki, Phys. Rev. E 75, 015203(R) (2007).

[7] A. B. Goryachev and A. V. Pokhilko, FEBS Lett. 582, 1437 (2008).

[8] Y. Mori, A. Jilkine, and L. Edelstein-Keshet, Biophys. J. 94, 3684 (2008).

[9] B. Rubinstein, B. D. Slaughter, and R. Li, Phys. Biol. 9, 045006 (2012).

[10] W. R. Holmes and L. Edelstein-Keshet, Phys. Biol. 13, 046001 (2016).
[11] J.-G. Chiou, S. A. Ramirez, T. C. Elston, T. P. Witelski, D. G. Schaeffer, and D. J. Lew, PLOS Comput. Biol. 14, e1006095 (2018).

[12] B. Jacobs, J. Molenaar, and E. E. Deinum, PLOS ONE 14, e0213188 (2019).

[13] F. Brauns, J. Halatek, and E. Frey, Phys. Rev. X 10, 041036 (2020).

[14] F. Brauns, H. Weyer, J. Halatek, J. Yoon, and E. Frey, Phys. Rev. Lett. 126, 104101 (2021).

[15] A. B. Goryachev and M. Leda, Cells 9, 2011 (2020).

[16] H. G. Othmer and E. Pate, Proc. Natl. Acad. Sci. USA 77, 4180 (1980).

[17] A. Hunding and P. Graae Sørensen, J. Math. Biol 26, 27 (1988).

[18] S. Ishihara and K. Kaneko, J. Theor. Biol. 238, 683 (2006).

[19] D. Ben-Zvi and N. Barkai, Proc. Natl. Acad. Sci. USA 107, 6924 (2010).

[20] O. Wartlick, P. Mumcu, A. Kicheva, T. Bittig, C. Seum, F. Jülicher, and M. González-Gaitán, Science 331, 1154 (2011). 
[21] D. M. Umulis and H. G. Othmer, Development 140, 4830 (2013).

[22] A. Onuki, Phase Transition Dynamics (Cambridge University Press, Cambridge, 2002).

[23] R. C. Desai and R. Kapral, Dynamics of Self-Organized and SelfAssembled Structures (Cambridge University Press, Cambridge, 2009).

[24] T. M. Rogers and R. C. Desai, Phy. Rev. B 39, 11956 (1989).

[25] D. Beysens, A. Steyer, P. Guenoun, D. Fritter, and C. M. Knobler, Phase Transit. 31, 219 (1991).

[26] J. H. Yao, K. R. Elder, H. Guo, and M. Grant, Phys. Rev. B 47, 14110 (1993).

[27] D. Beysens, C. R. Phys.Nucleation, 7, 1082 (2006).

[28] R. Khanna, N. K. Agnihotri, M. Vashishtha, A. Sharma, P. K. Jaiswal, and S. Puri, Phys. Rev. E 82, 011601 (2010).

[29] C. Narayanam, A. Kumar, S. Puri, and R. Khanna, Langmuir 33, 3341 (2017).

[30] G. Zhang, T. Yang, S. Yang, and Y. Wang, Phys. Rev. E 96, 032501 (2017).

[31] F. Bergmann, L. Rapp, and W. Zimmermann, Phys. Rev. E 98, 020603(R) (2018).

[32] H. Mori and Y. Kuramoto, Dissipative Structures and Chaos (Springer-Verlag, Berlin, 1998).

[33] U. Thiele, M. G. Velarde, and K. Neuffer, Phys. Rev. Lett. 87, 016104 (2001).

[34] U. Thiele, M. G. Velarde, K. Neuffer, and Y. Pomeau, Phys. Rev. E 64, 031602 (2001).

[35] E. Frey, J. Halatek, S. Kretschmer, and P. Schwille, in Physics of Biological Membranes (Springer International Publishing, Cham, 2018), pp. 229-260.

[36] J. Halatek and E. Frey, Nat. Phys. 14, 507 (2018).

[37] A. Novick-Cohen and L. A. Segel, Physica D 10, 277 (1984).

[38] See Supplemental Material at https://link.aps.org/supplemental/ 10.1103/PhysRevResearch.3.023198 for the pattern formation dynamics for parameter set A.

[39] C. Yeung, Phys. Rev. Lett. 61, 1135 (1988).

[40] P. Fratzl and J. L. Lebowitz, Acta metall 37, 3245 (1989).

[41] T. Koga, K. Kawasaki, M. Takenaka, and T. Hashimoto, Physica A 198, 473 (1993).

[42] H. Furukawa, J. Phys. Soc. Jpn. 58, 216 (1989).

[43] H. Tomita, Prog. Theor. Phys. 85, 47 (1991).

[44] T. Biancalani, F. Jafarpour, and N. Goldenfeld, Phys. Rev. Lett. 118, 018101 (2017).
[45] V. I. Kalikmanov, in Nucleation Theory, Lecture Notes in Physics, edited by V. Kalikmanov (Springer Netherlands, Dordrecht, 2013), pp. 17-41.

[46] T. Butler and N. Goldenfeld, Phys. Rev. E 80, 030902(R) (2009).

[47] T. Biancalani, D. Fanelli, and F. Di Patti, Phys. Rev. E 81, 046215 (2010).

[48] J. A. Bonachela, M. A. Muñoz, and S. A. Levin, J. Stat. Phys. 148, 724 (2012).

[49] T. Butler and N. Goldenfeld, Phys. Rev. E 84, 011112 (2011).

[50] D. Karig, K. M. Martini, T. Lu, N. A. DeLateur, N. Goldenfeld, and R. Weiss, Proc. Natl. Acad. Sci. USA 115, 6572 (2018).

[51] X. Guo, D.-J. Liu, and J. W. Evans, J. Chem. Phys. 130, 074106 (2009).

[52] C.-J. Wang, X. Guo, D.-J. Liu, and J. W. Evans, J. Stat. Phys. 144, 1308 (2011).

[53] A. S. Mikhailov, Foundations of Synergetics I: Distributed Active Systems, Vol. 51 (Springer-Verlag, Berlin, 1990).

[54] D. Zwicker, A. A. Hyman, and F. Jülicher, Phys. Rev. E 92, 012317 (2015).

[55] T. Frohoff-Hülsmann, J. Wrembel, and U. Thiele, Phys. Rev. E 103, 042602 (2021).

[56] A. P. Solon, J. Stenhammar, M. E. Cates, Y. Kafri, and J. Tailleur, New J. Phys. 20, 075001 (2018).

[57] G. Gompper, R. G. Winkler, T. Speck, A. Solon, F. Nardini, C.and Peruani, H. Löwen, R. Golestanian, U. B. Kaupp, L. Alvarez et al., J. Phys.: Condens. Matter 32, 193001 (2020).

[58] X. Chen, X. Wu, H. Wu, and M. Zhang, Nat. Neurosci. 23, 301 (2020).

[59] S. D. Hansen, W. Y. C. Huang, Y. K. Lee, P. Bieling, S. M. Christensen, and J. T. Groves, Proc. Natl. Acad. Sci. USA 116, 15013 (2019).

[60] A. A. Hyman, C. A. Weber, and F. Jülicher, Annu. Rev. Cell Dev. Biol. 30, 39 (2014).

[61] C. P. Brangwynne, P. Tompa, and R. V. Pappu, Nat. Phys. 11, 899 (2015).

[62] S. Boeynaems, S. Alberti, N. L. Fawzi, T. Mittag, M. Polymenidou, F. Rousseau, J. Schymkowitz, J. Shorter, B. Wolozin, L. Van Den Bosch et al., Trends Cell Biol. 28, 420 (2018).

[63] J. Berry, C. P. Brangwynne, and M. Haataja, Rep. Prog. Phys. 81, 046601 (2018).

[64] Y. Morita and T. Ogawa, Nonlinearity 23, 1387 (2010). 